\title{
RÉPERTOIRE SYSTÉMATIQUE \\ DES NOMS DE GENRES DE VERTÉBRÉS \\ (suite)
}

Par Yves J. GOLVAN

Cabalus HUTTON 1874: syn. de Huttonena MATHEWS 1929.

Cabassous Mc MURTRIE 1831 : M.EDENT. (Xenarthr.) Dasypodid. (Dasypodin.).

Cabdio HAMILTON - BUCHANAN 1822: P.-CYPRINIF. (Cyprinoid.) Cyprinid.

Cabotia de BUEN 1930 : syn. de Cabotichthys WHITLEY 1940.

Cabotichthys WHITLEY 1940 : P.PERCIF. (Gobioid.) Eleotrid.

Cabotina : syn. de Fagea de BUEN 1940.

Cabrita GRAY 1838 : R.-SQUAMAT. (Saur.) Lacertid.

Cabritopsis BEDDOME $1870:$ R.SQUAMAT. (Saur.) Lacertid.

Cacajao LESSON 1840 : M.-PRIMAT. (Simioid.) Cebid. (Pithecin.).

Cacatua VIEILLOT 1817 : syn. de Kakatoë CUVIER 1800.

Caccabis KAUP 1829 : syn. d'Alectoris KAUP 1829.

Cachius GUNTHER 1868: P.-CYPRINIF. (Cyprinoid.) Cyprinid.

Cachryx COPE 1866 : R.-SQUAMAT. (Saur.) Iguanid.

Cachuga GRAY 1855 : R.-TESTUDIN. (Thecoph.) Emydid.

Cacicus de LACEPEDE 1799: Av. PASSERIF. (Acromyod.) Icterid. (Cacicin.).

Cacodoxus CANTOR 1850 : P.-PERCIF. (Percoid.) Chaetodontid.

Cacomantis MULLER 1842: Av. CUCULLIF. Cucullid.

Cacophryne DAVIS 1835: Amph. ANOUR. Brachycephalid.

Cacopitta BONAPARTE 1850: Av. PASSERIF. (Mesomyod.) Pittid.

Cacopoides BARBOUR 1908: syn. de Kaloula GRAY 1831.

Cacopus GUNTHER 1864: syn. d'Uperodon DUMERIL et BIBRON 1841.

Cacosternum BOULENGER 1887 : Amph. ANOUR. Microhylid.

Cactornis GOULD 1837: Av. PASSERIF. (Acromyod.) Fringillid. (Geospizin.).

Cactospiza RIDGWAY 1897: Av. PASSERIF. (Acromyod.) Fringillid. (Geospizin.).

Cacumen WHITLEY 1931 : P.-PERCIF. (Scorpaenoid.) Platycephalid.

Cadea GRAY 1844 : R.-SQUAMAT. (Saur.) Amphisbaenid.

Caecilia LINNE 1758: Amph. APOD. Caeciliid.

Caeciliophis DUMERIL 1856 : errat. pro Coeciliophis KAUP 1856.

Caecobarbus BOULENGER 1921: P.-CYPRINIF. (Cyprinoid.) Cyprinid.

Caecomastacembelus POLL 1959 : P. - MASTACEMBELIF. Mastacembelid.

Caecorhamdella BORODIN 1927 : P.-CYPRINIF. (Siluroid.) Bagrid. 
Caecorhamdia NORMAN 1926 : P.CYPRINIF. (Siluroid.) Bagrid.

Caecula VAHL 1794: P.-ANGUILLIF. (Congroid.) Ophichthyid.

Caenolestes THOMAS 1895: M.MARSUP. Caenolestid.

Caenotriccus SCLATER 1888: Av. PASSERIF. (Mesomyod.) Tyrannid.

Caenotronus GUNTHER 1864 : P.CYPRINIF, (Characoid.) Characid.

Caeruleus : P.-CLUPEIF. (Clupeoid.) Clupeid.

Caesio de LACEPEDE 1802: P.PERCIF. (Percoid.) Lutjanid. (ou Caesioid.).

Caesiomorus de LACEPEDE 1802 : syn. de Glaucus GOUAN 1770.

Caesioperca CASTELNAU 1872: P.-PERCIF. (Percoid.) Serranid.

Caesioscorpis WHITLEY 1945 : P.PERCIF. (Percoid.) Lutjanid.

Caesiosoma KAUP 1863 : P.-PERCIF. (Percoid.) Chaetodontid.

Caeso GISTEL 1848 : P.-PERCIF. (Percoid.) Sparid.

Caffrapus ROBERTS 1922: Av. APODIF. Apodid.

Caica LESSON 1830 : Av. PSITTACIF. Psittacid.

Caigator DERANYXIAGALA 1947 : R.-LORICAT. Alligatorid.

Caiman SPIX 1825 : R.-LORICAT. Alligatorid.

Cainodactylus BARBOUR 1924: R.SQUAMAT. (Saur.) Geckonid.

Cainosilurus Mc LEAY 1881: $P$.CYPRINIF. (Siluroid.) Plotosid.

Cairina FLEMING 1822: Av. ANATIF. Anatid. (Anatin.).

Calabaria GRAY 1858: R.-SQUA$M A T$. (Serpent.) Pythonid.

Calamanthella SWINHOE 1859 : Av. PASSERIF. (Acromyod.) Timaliid.

Calamanthus GOULD 1838: Av. PASSERIF. (Acromyod.) Timaliid.

Calamaria BOIE 1826 : R.-SQUAMAT. (Serpent.) Colubrid.
Calamelaps GUNTHER 1866 : R.SQUAMAT. (Serpent.) Boigid.

Calamiana HERRE 1945 : P.-PERCIF. (Gobioid.) Gobiid.

Calamobates de WITTE 1930 : Amph. ANOUR. Microhylid.

Calamocichla SHARPE 1883: Av. PASSERIF. (Acromyod.) Sylviid.

Calamodon AFRANIO do AMARAL 1935 : R.-SQUAMAT. (Serpent.) Boigid.

Calamodus KAUP 1829: syn. d'Acrocephalus NAUMANN 1804.

Calamodyta KAUP 1829 : syn. d'Acrocephalus NAUMANN 1804.

Calamoecetor SCLATER 1936: Av. PASSERIF. (Acromyod.) Turdid.

Calamoherpe BOIE 1822: syn. d'Acrocephalus NAUMANN 1804.

Calamoichthys SMITH 1866: P.POLYPTERIF. Polypterid.

Calamonastes SHARPE 1883: Av. PASSERIF. (Acromyod.) Timaliid.

Calamonastides GRANT et MACKWORTH 1940 : Av. PASSERIF. (Acromyod.) Muscicapid.

Calamornis SCLATER 1927: Av. PASSERIF. (Acromyod.) Sylviid.

Calamorhabdium BOETTEGER 1898 : R.-SQUAMAT. (Serpent.) Colubrid.

Calamorhamphus : Av. PICIF. Bucconid.

Calamospiza BONAPARTE 1838 : Av. PASSERIF. (Acromyod.) Icterid. (Agelaein.).

Calamuraena WHITLEY 1944 : P.$A N G U I L L I F . \quad$ (Congroid.) Ophichthyid.

Calamus SWAINSON 1839: P.-PERCIF. (Percoid.) Sparid.

Calandra ZIMMERMANN 1793 : Av. PASSERIF. (Acromyod.) Alaudid.

Calandrella KAUP 1829 : Av. PAS. SERIF. (Acromyod.) Alaudid.

Calandria des MURS 1856 : syn. de Calandra ZIMMERMANN 1793. 
Calandritis CABANIS 1851 : emend. pro Calandrella KAUP 1829.

Calandula : vide Calendula SWAINSON 1837.

Calastrapia SHARPE 1898: Av. PASSERIF. (Acromyod.) Paradiseid. (Paradisein.).

Calcarius BECHSTEIN 1802: Av. PASSERIF. (Acromyod.) Fringillid. (Emberizin.).

Calcarobrotula FOWLER 1946 : P.PERCIF. (Ophidioid.) Brotulid.

Calchostetha CABANIS 1851: Av. PASSERIF. (Acromyod.) Nectariniid.

Calcochloris MIVART 1867: syn. d'Amblysomus POMEL 1848.

Caleleotris : vide Calleleotris GILL 1863.

Calendula SWAINSON 1837: Av. PASSERIF. (Acromyod.) Alaudid.

Calendulauda BLYTH 1855: Av. PASSERIF. (Acromyod.) Alaudid.

Calherodius BONAPARTE 1855 : Av. ARDEIF. Ardeid.

Calicalcus BONAPARTE 1854: Av. PASSERIF. (Acromyod.) Vangid.

Calictis GRAY 1864 : syn. d'Herpestes ILLIGER 1811.

Calidris ILLIGER 1811: Av. CHARADRIIF. Charadritd. (Scolopacin.).

Callacanthis REICHENBACH 1850 : Av. PASSERIF. (Acromyod.) Fringillid. (Fringillin.).

Callaeas FORSTER 1788 : Av. PASSERIF. (Acromyod.) Corvid.

Callaeops OGILVIE-GRANT 1895 : Av. PASSERIF. (Acromyod.) Corvid.

Callagur GRAY 1870: R.-TESTUDIN. (Thecoph.) Emydid.

Callanthias LOWE 1839 : P.-PERCIF. (Percoid.) Serranid.

Callaus JORDAN 1889 : P.-PERCIF. (Percoid.) Sciaenid.

Callechelys KAUP 1856: P.-ANGUILLIF. (Congroid.) Ophichthyid.

Calleleotris GILL 1863: P.-PERCIF. (Gobioid.) Eleotrid.
Callene BLYTH 1847 : Av. PASSERIF. (Acromyod.) Turdid. (ou Timaliid.).

Calliblennius BARBOUR 1912 : P.PERCIF. (Blennioid.) Blenniid.

Callicanthus $S$ W A I N S O N 1839: P. - PERCIF. (Acanthuroid.) Acanthurid.

Callicebus THOMAS 1903 : M.-PRIMAT. (Simioid.) Cebid. (Callicebin.).

Callichelys GRAY 1863 : R.-TESTUDIN. (Thecoph.) Emydid.

Callichrous HAMILTON-BUCHANAN 1822 : P.-CYPRINIF. (Siluroid.) Silurid.

Callichthys GRONOV 1763 : P.-CYPRINIF. (Siluroid.) Callichthyid.

Calliclinus GILL 1860 : P.-PERCIF. (Blennioid.) Clinid.

Callidulus FOWLER 1907 : P.-PERCIF. (Percoid.) Serranid.

Calliechthrus CABANIS et HEINE : Av. CUCULLIF. Cucullid.

Callieleotris FOWLER 1934: syn. de Borodamirus WH I TLEY 1935.

Calligenia $M U L S A N T 1876$ : Av. APODIF. Trochilid.

Calliglutus BARDOUR et NOBLE 1916: Amph. ANOUR. Microhylid.

Callignathula STRAND 1928 : syn. de Kogia GRAY 1846.

Callignathus GILL 1871: syn. de Kogia GRAY 1846.

Callimico RIBEIRO 1911 : M.-PRIMAT. (Simioid.) Hapalid. (Callimicoin.).

Callimucenus WHITLEY 1934 : P.PERCIF. (Callionymoid.) Callionymid.

Callinycteris JENTINK 1898 : syn. d'Eonycteris DOBSON 1873.

Calliodon GRAY 1871 : syn. de Mesoplodon GERVAIS 1850.

Callionymus LINNE 1758 : P.-PERCIF. (Callionymoid.) Callionymid.

Calliope GOULD 1836 : Av. PASSERIF. (Acromyod.) Turdid.

Calliophis GRAY 1834: R.-SQUAMAT. (Serpent.) Elapid. 
Callipepla WA GLER 1832: Av. PHASIANIF. Phasianid.

Callipharus ELLIOT 1879: Av. APODIF. Trochilid.

Calliphlox BOIE 1831: Av. APODIF. Trochilid.

Calliptilodes STRAND 1928: Av. PSITTACIF. Psittacid.

Calliptilus SUNDEVALL 1872 : syn. de Calliptilodes STRAND 1928.

Callirhinus BLANCHARD 1850 : errat. pro Callorhinus GRAY 1859.

Callisaurus : errat. pro Callysaurus de BLAINVILLE 1835.

Calliscyllium TANAKA 1912: P.GALEIF. (Carcharhinoid.) Scyliorhinid.

Callisitta BONAPARTE 1850: Av. PASSERIF. (Acromyod.) Sittid.

Calliste BOIE 1826 : syn. de Calospiza GRAY 1840.

Callistus LAFRESNAYE 1843: Av. PASSERIF. (Acromyod.) Tanagrid.

Callithrix GEOFFROY ST-HILAIRE 1812 : syn. de Callicebus THOMAS 1903.

Callithrix ERXLEBEN 1777 : syn. de Hapale ILLIGER 1811.

Calliurichthys JORDAN et FOWLER 1903 : P.-PERCIF. (Callio nymoid.) Callionymid.

Calliurus RAFINESQUE 1818 : P.PERCIF. (Percoid.) Centrarchid.

Callixalus : Amph. ANOUR. Rhaco. phorid.

Callocephalon LESSON 1837: Av. PSITTACIF. Psittacid.

Callocephalus CUVIER 1824: syn. de Phoca LINNE 1758.

Callochromis RE GAN 1920 : P.PERCIF. (Percoid.) Cichlid.

Callogobius BLEEKER 1874 : P.PERCIF. (Gobioid.) Gobiid.

Callomystax GUNTHER 1864 : P.CYPRINIF. (Siluroid.) Bagrid.

Callopanchax MYERS 1933 : P.-CYPRINODONTIF. Cyprinodontid.

Callopeltis BONAPARTE 1841: syn. de Coluber LINNE 1758.
Callophis GUNTHER 1859 : errat. pro Calliophis GRAY 1834.

Callophysus MULLER et TROSCHEL 1849 : P.-CYPRINIF. (Siluroid.) Bagrid.

Callopistes GRAVENHORST 1838: R.-SQUAMAT. (Saur.) Tejid.

Callopistria AFRANIO do AMARAL 1935 : R.-SQUAMAT. (Serpent.) Boigid.

Calloplesiops FOWLER et BEAN 1930 : P.-PERCIF. (Percoid.) Plesiopid.

Calloptilum RICHARDSON 1845 : P.-GADIF. Gadid.

Callorhabdiun : R.-SQUAMAT. (Serpent.) Colubrid.

Callorhinus GRAY 1859 : M.-PINNIP. Otariid. (Arctocephalin.).

Callorhynchus GRONOV 1763: P.-HOLOCEPH. Callorhynchid.

Callosciurus GRAY 1867: M.-RODENT. (Sciuromorph.) Sciurid. (Sciurin.).

Callositta : errat. pro Ca lo c it t a GRAY 1841.

Callopsermophilus MERRIAM 1897: s./g. de Citellus OKEN 1816.

Callotaria PALMER 1892 : syn. de Callorhinus GRAY 1859.

Calluela STOLICZKA 1872 : Amph. ANOUR. Microhylid.

Callula GUNTHER 1864: Amph. ANOUR. Microhylid.

Callulops B O U L E N G E R 1888: Amph. ANOUR. Microhylid.

Callyodon GRONOV 1763 : P.-PERCIF. (Labroid.) Scarid. (ou Callyodontid.).

Callyodonichthys BLEEKER 1861 : P.-PERCIF. (Labroid.) Scarid.

Callysaurus de BLAINVILLE 1835 : R.-SQUAMAT. (Saur.) Iguanid.

Caloardea ROBERTS 1926: syn. de Calherodius BONAPARTE 1855 .

Calobates KAUP 1829: s./g. de Motacilla LINNE 1758.

Calochaetes SCLATER 1879: Av. PASSERIF. (Acromyod.) Tanagrid.

Calochelys FRITSCH 1879: syn. de Callichelys GRAY 1863.

ANN. de Parasitologie, T. XXXVI, No 5-6. -1961. 
Calocitta GRAY 1841: Av. PASSERIF. (Acromyod.) Corvid.

Calodactylodes STRAND 1928 : R.SQUAMAT. (Saur.) Geckonid.

Calodactylus BEDDOME 1870 : syn. de Calodactylodes STRAND 1928.

Calodromas SCLATER et SALVIN 1873: Av. TINAMIF. Tinamid.

Caloenas GRAY 1840 : Av. COLUMBIF. Calumbid. (Calumbin.).

Calogale GRAY 1864: syn. d'Herpestes ILLIGER 1811.

Calomys WATERHOUSE 1837: syn. d'Hesperomys WATERHOUSE 1839.

Calomyscus THOMAS 1905: M.RODENT. (Myomorph.) Cricetid. (Cricetin.).

Calonectris MATHEWS et IREDALE 1915: Av. PROCELLARIF. Procellariid.

Calopelia SALVADORI 1893: Av. COLUMBIF. Columbid. (Columbin.).

Caloperdix BLYTH 1861 : Av. PHASIANIF. Phasianid. (Phasianin.).

Calopeza RIDGWAY 1885 : Av. TINAMIF. Tinamid.

Calophasis ELLIOT 1872: Av. PHASIANIF. Phasianid.

Calophryne TSCHUDI 1838: Amph. ANOUR. Microhylid.

Calophysus MULLER et TROSCHEL 1842 : vide Callophysus M. et T. 1842 .

Calopitta SCLATER 1888: Av. PASSERIF. (Mesomyod.) Pittid.

Caloprymnus THOMAS 1888: M.MARSUP. Macropodid. (Potoroin.).

Calopsittacus SALVIN 1882: Av. PSITTACIF. Psittacid.

Calopterocles ROBERTS 1922: syn. de Pterocles TEMMINCK 1815.

Calopus : R.-SQUAMAT. (Saur.) Geckonid.

Calorhamphus (LESSON 1839) STRICKLAND 1842: Av. PICIF. Capitonid.

Calornis GRAY 1841: Av. PASSERIF. (Acromyod.) Sturnid. (Graculin.).
Calositta des MURS 1854: errat. pro Calocitta GRAY 1841.

Calospiza GRAY 1840 : Av. PASSERIF. (Acromyod.) Tanagrid.

Calotes CUVIER 1817: R.-SQUAMAT. (Saur.) Agamid.

Calothorax GRAY 1840: syn. de Lafresnayea BONAPARTE 1850 .

Calotomus G I L B E R T 1890 : P.PERCIF. (Labroid.) Scarid.

Calotreron HEINE 1890 : Av. COLUMBIF. Columbid. (Treronin.).

Calpata STRAND 1928: syn. de Saundersilarus DWIGHT 1926.

Caluromys ALLEN 1900 : syn. de Philander BRISSON 1762.

Caluromysiops SANBORN 1951: M. MARSUP. Didelphid.

Calycilonidotus AYRES 1854 : P.PERCIF. (Cottoid.) Cottid.

Calymmichthys JORDAN et THOMPSON 1914 : P.-PERCIF. (Callionymoid.) Callionymid.

Calypte GOULD 1861: Av. APODIF. Trochilid.

Calyptocephalella STRAND 1928 : Amph. ANOUR. Leptodactylid.

Calyptocephalus DUMERIL et BIBRON 1841: Amph. ANOUR. Leptodactylid.

Calyptocichla OBERHOLSER 1905 : Av. PASSERIF. (Acromyod.) Pycnonotid.

Calyptomena RAFFLES 1822: Av. PASSERIF. (Desmodactyl.) Eurylaimid.

Calyptophilus CORY 1884: Av. PASSERIF. (Acromyod.) Tanagrid.

Calyptorhynchus VIGORS 1825: Av. PSITTACIF. Psittacid.

Calyptura SWAINSON 1832: Av. PASSERIF. (Mesomyod.) Cotingid.

Camarhynchus GOULD 1837 : Av. PASSERIF. (Acromyod.) Fringillid. (Geospizin.).

Camaroptera SUNDEVALL 1850 : Av. PASSERIF. (Acromyod.) Sylviid.

Camelus LINNE 1758: M.-PARAX. (Solenodont.) Camelid. 
Camnbellina FOWLER 1958: P.BERYCIF. Diretmid.

Campephaga VIEILLOT 1816: Av. PASSERIF. (Acromyod.) Campephagid.

Campephilus GRAY 1840 : Av. PICIF. Picid. (Picin.).

Campethera GRAY 1841: Av. PICIF. Picid. (Picin.).

Campichthys WHITLEY 1931 : P.SYNGNATHIF. Syngnathid.

Campicola SCHULTZ 1890: syn. de Campicolina STRAND 1928.

Campicolina STRAND 1928: s./g. d'Arvicola de LACEPEDE 1799.

Campochaera SHARPE 1878: Av. PASSERIF. (Acromyod.) Campephagid.

Campogramma REGAN 1903 : P. PERCIF. (Percoid.) Carangid.

Campophilus AGASSIZ 1846: emend. pro Campephilus GRAY 1840.

Camposella FERNANDEZ-YEPEZ : syn. de Bertelsenna WHITLEY 1954.

Camposella COLE 1919 : P.-CYPRINIF. (Characoid.) Characid.

Camposichthys TRAVASSOS 1946 : P.-CYPRINIF. (Characoid.) Characid.

Camposichthys WHITLEY 1953 : syn. Stupens WHITLEY 1954.

Campostoma AGASSIZ 1855: P.CYPRINIF. (Cyprinoid.) Cyprinid.

Campothera AGASSIZ 1846 : emend. pro Campethera GRAY 1841.

Camptorhynchus BONAPARTE 1838: Av. ANATIF. Anatid. (Anatin.).

Camptostoma SCLATER 1857: Av. PASSERIF. (Mesomyod.) Tyrannid.

Camptotaemus GRAY: Av. ANATIF. Anatid. (Anatin.).

Campylodon CUVIER et VALENCIENNES 1832 : P.-NOTACANTHIF. Notacanthid.

Campylomormyrus $B L E E K E R$ 1874: P.-CLUPEIF. (Mormyroid.) Mormyrid.

Campylopterus SWAINSON 1827 : Av. APODIF. Trochilid.
Campylorhamphus BERTONI 1901: Av. PASSERIF. (Mesomyod.) Dendrocolaptid.

Campylorhynchus SPIX 1824: Av. PASSERIF. (Acromyod.) Troglodytid.

Canachites STEJNEGER 1885: Av. PHASIANIF. Tetraonid.

Cancroma LINNE 1758: Av. ARDEIF. Ardeid.

Candidia JORDAN et RICHARDSON 1909 : P.-CYPRINIF. (Cyprinoid.) Cyprinid.

Candoia GRAY 1842: R.-SQUAMAT. (Serpent.) Boid.

Caninoa NARDO 1841: syn. de Squalus LINNE 1758.

Caninotus : P.-GALEIF. (Carcharhinoid.) Scyliorhinid.

Canirallus BONAPARTE 1856:Av. RALLIF. Rallid.

Canis LIN NE 1758: M.-FISSIP. Canid. (Canin.).

Cannabina GRAY 1840: syn. de Carduelis BRISSON 1760.

Cannomvs THOMAS 1915: M.-RODENT. (Hystricomorph.) Rhizomyid.

Cannorhynchus CANTOR 1850 : P.-AULOSTOMIF. Fistulariid.

Cansumys ALLEN 1928: syn. de Cricetulus MILNE-EDWARDS 1867.

Cantharus CUVIER 1817 : syn. de Cantharusa STRAND 1928.

Cantharusa STRAND 1928: P.PERCIF. (Percoid.) Sparid.

Cantherhines SWAINSON 1839: syn. d'Amanses GRAY 1875.

Canthidermis SWAINSON 1839: P.-PERCIF, (Balistoid.) Balistid.

Canthigaster SWAINSON 1839: P.-TETRAODONTIF. (Ostracionoid.) Canthigasterid.

Canthogaster BLEEKER 1870: syn. de Canthigaster SWAINSON 1839.

Canthophrys SWAINSON 1838: P.-CYPRINIF. (Cyprinoid.) Cobitid.

Canthopomus EIGENMANN 1942: P.-CYPRINIF. (Siluroid.) Loricariid. 
Canthyrhynchus (SWAINSON 1839) AGASSIZ 1846 : P.PERCIF. (Cottoid.) Agonid.

Cantoria GIRARD 1857 : R.-SQUAMAT. (Serpent.) Boigid.

Cantoria KAUP 1858 : P.-PLEURONECTIF. (Soleoid.) Cynoglossid.

Cantorusia WHITLEY 1940 : P.PLEURONECTIF. (Soleoid.) Cynoglossid.

Canutus BREHM 1830 : syn. de Calidris ILLIGER 1811.

Capaccinus BONAPARTE 1841: syn. de Myotis KAUP 1829.

Capartella : CHABANAUD 1950 : P.-PLEURONECTIF. (Soleoid.) Soleid.

Capella FRENZEL 1801 : Av. CHARADRIIF. Charadriid. (Scolopacin.).

Capella KEYSERLING et BLASIUS 1840 : syn. de Rupicapra de BLAINVILLE 1816.

Capito VIEILLOT 1816 : Av. PICIF. Capitonid.

Capoeta CUVIER et VALENCIENNES 1842 : P.-CYPRINIF. (Cyprinoid.) Cyprinid.

Capoetobrama BERG 1916 : P.-CYPRINIF. (Cyprinoid.) Cyprinid.

Capra LINNE 1758: M.-PARAX. (Solenodont.) Bovid. (Caprin.).

Caprea OGILBY 1837 : syn. de Capreolus GRAY 1821.

Capreolus GRAY 1821: M.-PARAX. (Solenodont.) Cervid.

Capreolus FRISCH 1775: nom. nud. pro Capreolus GRAY 1821.

Capricornis OGILBY 1837 : M.-PARAX. (Solenodont.) Bovid. (Rupicaprin.).

Capricornulus HEUDE $1898: s . / g$. de Capricornis OGILBY 1837.

Capricornus GRAY 1862: errat. pro Capricornis OGILBY 1837.

Capridoglanis : errat. pro Copidoglanis GUNTHER 1864.

Caprimulgus LINNE 1758: Av. CAPRIMULGIF. Caprimulgid. (Caprimulgin.).

Caprina WAGNER 1844: syn. de Naemorhedus SMITH 1827.
Caprios WAGLER 1830 : syn. de Desmana GULDENSTAEDT 1777.

Caprisculus STRAND 1928: syn. d'Ovis LINNE 1758.

Capriscus GL OGER 1841: syn. d'Ovis LINNE 1758.

Capriscus SCHAEFFER 1760 : P.PERCIF. (Balistoid.) Balistid.

Caprodon TEMMINCK et SCHLEGEL 1843 : P.-PERCIF. (Percoid.) Serranid.

Caprolagus BLYTH 1845: M.-LAGOMORPH. Leporid. (Leporin.).

Capromys D E S M R E S T 1822: M.-RODENT. (Caviomorph.) Capromyid.

Caprophonus MULLER et TROSCHEL 1847 : P.-ZEIF. Caproid.

Capros de LACEPEDE 1802: P.-ZEIF. Caproid.

Caprovis HODGSON 1847: syn. d'Ovis LINNE 1758.

Caprupeneus WHITLEY 1931 : P.PERCIF. (Percoid.) Mullid.

Capsiempis CABANIS et HEINE 1859: Av. PASSERIF. (Mesomyod.) Tyrannid. (Spiziornin.).

Caquetaia FOWLER 1945 : P.-PERCIF. (Percoid.) Cichlid.

Caracal GRAY 1843 : s./g. de Felis LINNE 1758.

Caracanthus KROYER 1844 : P.PERCIF. (Scorpaenoid.) Scorpaenid.

Caracara MERREM 1826 : syn. de Polyborus VIEILLOT 1816.

Caragobioides SMITH 1945: P.PERCIF. (Gobioid.) Gobiid.

Caragobius JORDAN et SEALE 1906 : P.-PERCIF. (Gobioid.) Gobiid.

Caragola GRAY 1851 : Cyclost. PETROMYZONIF. Geotriidae.

Caralophia BOHLKE 1955 : P.-ANGUILLIF. (Congroid.) Ophichthyid.

Caranactis REGAN et TREWAVAS 1933 : P.-LOPHIIF. (Ceratoid.) Diceratiid.

Carangichthys BLEEKER 1870 : P.-PERCIF. (Percoid.) Carangid. 
Carangoides $B L E E K E R \quad 1855$ : P.-PERCIF. (Percoid.) Carangid.

Carangops GILL 1862 : P.-PERCIF. (Percoid.) Carangid.

Carangus GRIFFITH 1834: P.-PERCIF. (Percoid.) Carangid.

Caranthus B A R N A R D 1927 : P.PERCIF. (Percoid.) Sparid.

Caranx de LACEPEDE 1802 : P.-PERCIF. (Percoid.) Carangid.

Caranxomorus de LA C E P E D E 1802: P.-PERCIF. (Percoid.) Lutjanid.

Carapo OKEN 1817 : syn. de Gymnotus LINNE 1758.

Carapus RAFINESQUE 1810 : P.CYPRINIF. (Gymnotoid.) Gymnotid.

Carapus CUVIER 1817: syn. de Fierasfer OKEN 1817.

Caraspius NICHOLS 1925 : P.-CYPRINIF. (Cyprinoid.) Cyprinid.

Carassioides OSHIMA 1926 : $P$.- CYPRINIF. (Cyprinoid.) Cyprinid.

Carassiops OGILBY 1897: P.-MUGILIF. Atherinid.

Carassiops : P.-PERCIF. (Gobioid.) Eleotrid.

Carassius JAROCKI 1822 : P.-CYPRINIF. (Cyprinoid.) Cyprinid.

Carbo de LACEPEDE 1799: syn. de Phalacrocorax BRISSON 1760.

Carcharhinus de BLAINVILLE 1816 : syn. de Carcharias RAFINESQUE 1810 ou bon genre [P.-GALEIF. (Carcharhinoid.) Carcharhinid.] si Carcharias RAFINESQUE 1810 est syn. d'Odontaspis AGASSIZ 1835.

Carcharias RAFINESQUE 1810 : P. - GALEIF. (Carcharhinoid.) Carcharhinid.

Carcharias CUVIER 1812 : syn. de Carcharias RAFINESQUE 1810.

Carcharocles JORDAN 1923: P.-GALEIF. (Isuroid.) Isurid.

Carcharodon (SMITH) MULLER et HENLE 1838: P.-GALEIF. (Isuroid.) Isurid.

Carcineutes CABANIS et HEINE 1860: syn. de Lacedo REICHENBACH 1851.
Cardellina de BUS 1849 : Av. PASSERIF. (Acromyod.) Parulid.

Cardinalis BONAPARTE 1831: syn. de Richemondena MATHEWS et IREDALE 1918.

Cardinella : errat. pro Cardellina du BUS 1849.

Cardiocranus SATUNIN 1903 : M.RODENT. (Myomorph.) Dipodid. (Cardiocranin.).

Cardioderma PETERS $1873: s . / g$. de Megaderma GE OF $F \mathrm{R} Y$ St-HILAIRE 1810.

Cardioglossa BOULENGER 1900 : Amph. ANOUR. Ranid.

Cardionharynx $P O L L 1942$ : $P$.PERCIF. (Percoid.) Cichlid.

Carduelis $B R I S S O N$ 1760: Av. PASSERIF. (Acromyod.) Fringillid. (Fringillin.).

Carduiceps CLAY et MEINERTZTRAGEN 1939: Av. CHARADRIIF. Charadriid. (Scolopacin.).

Careliparis GARMAN 1892: P.-PERClF. (Cottoid.) Cyclopterid.

Caremitra JORDAN et EVERMANN 1897 : P. - PERCIF. (Cottoid.) Cyclopterid.

Careproctus KROYER 1861: syn. de Gymnolycodes VAILLANT 1888.

Caretta RAFINESQUE 1814: R.TESTUDIN. (Thecoph.) Cheloniid.

Carettochelys RAMSAY 1886: R.TESTUDIN. (Thecoph.) Carettochelyid.

Cariama BRUNNICH 1772: Av. FALCONIF. Falconid. (Milvin.).

Cariama BRISSON 1760 : Av. RALLIF. Cariamid.

Caribhregma BREDER 1927: syn. de Pseudogramma BLEEKER 1875.

Cariburus PARR 1946 : P.-GADIF. Macrurid.

Carina S.D.W. 1836 : Cairina FLEMING 1822 .

Carine KAUP 1829 : syn. d'Athene BOIE 1822.

Carinotus LAMONTE $1933: s . / g$. de Plecostomus GRONOV 1763.

Caristioides WHITLEY 1948: 
P.-PERCIF. (Stromateoid.) Stromateid.

Caristius GILL et SMITH 1905: P.-BERYCIF. Caristiid.

Carlana STRAND 1928 : P.-CYPRINIF. (Characoid.) Characid.

Carlia MEEK 1914 : syn. de Carlana STRAND 1928.

Carlhubbsia WHITLEY 1953 : P.CYPRINODONTIF. Poeciliid.

Carnegiella EIGENMANN 1909 : P.-CYPRINIF, (Characoid.) Characid.

Carollia GRAY 1838 : M.-CHIROPT. (Microchiropt.) Phyllostomatid. (Carolliin.).

Carphibis REICHENBACH 1852 : Av. ARDEIF. Plataleid. (Threskiornithin.).

Carphophiops GERVAIS 1849 : R.SQUAMAT. (Serpent.) Colubrid.

Carphophis G ER V A I S 1849 : R.SQUAMAT. (Serpent.) Colubrid.

Carpio HECKEL 1843 : P.-CYPRINIF. (Cyprinoid.) Cyprinid.

Carpiodes RAFINESQUE 1820 : P.CYPRINIF. (Cyprinoid.) Catostomid.

Carpionichthys BLEEKER 1862: P.-CYPRINIF. (Cyprinoid.) Cyprinid.

Carpitalpa LUNDHOLM $1955: s . / g$. de Chlorotalpa ROBERTS 1924 .

Carpococcyx GRAY 1840 : Av. CUCULLIF. Cucullid.

Carpodacus KAUP 1829 : Av. PASSERIF. (Acromyod.) Fringillid. (Fringillin.).

Carpodectes SALVIN 1865: Av. PASSERIF. (Mesomyod.) Cotingid.

Carpomys THOMAS 1895 : M.-RODENT. (Myomorph.) Murid. (Murin.).

Carponycteris LYDEKKER 1891: syn. de Macroglossus CUVIER 1824.

Carpophaga SELBY 1835 : syn. de Carpophagella STRAND 1928.

Carpophaga BILLBERG 1828: Av. COLUMBIF. Columbid. (Treronin.).
Carpophagella STRAND 1928 : Av. COLUMBIF. Columbid. (Treronin.).

Carpophis DUNN 1853: syn. de Carphophis GERVAIS 1849.

Carpospixa von MULLER 1854 : Av. PASSERIF. (Acromyod.) Ploceid.

Carterodon WATERHOUSE 1848 : M.-RODENT. (Caviomorph.) Echimyid. (Echimyin.).

Carterornis MATHEWS 1912 : syn. de Monarcha VIGORS et HORSFIELD 1826.

Caryomys THOMAS 1911 : syn. de Clethrionomys TILESIUS 1850.

Caryothraustes REICHENBACH 1850: Av. PASSERIF. (Acromyod.) Fringillid. (Coccothraustin.).

Casarca BONAPARTE 1838: Av. ANATIF. Anatid. (Anatin.).

Casarea GRAY 1842 : R.-SQUAMAT. (Serpent.) Boid.

Cascadura ELLIS 1913 : P.-CYPRINIF. (Siluroid.) Callichthyid.

Casinycteris TH OMAS 1910 : M.CHIROPT. (Megachiropt.) Pteropid. (Pteropin.).

Casiornis des MURS 1855: Av. PASSERIF. (Mesomyod.) Cotingid.

Casmerodius GLOGER 1842: Av. ARDEIF. Ardeid.

Caspedoprion HARTERT 1902: errat. pro Craspedoprion BERLEPSCH et HARTERT 1902.

Caspialosa BERG 1916: P.-CLUPEIF. (Clupeoid.) Clupeid.

Caspiomyzon BERG 1906 : Cyclost. PETROMYZONIF. Petromyzonid.

Caspiosoma ILJIN 1927 : P.-PERCIF. (Gobioid.) Gobiid.

Cassiculus SWAINSON 1827: Av. PASSERIF. (Acromyod.) Icterid. (Cacicin.).

Cassicus ILLIGER 1811: syn. de Cacicus de LACEPEDE 1799.

Cassidix LESSON 1831 : Av. PASSERIF. (Acromyod.) Icterid. (Cacicin.). 
Cassigobius WHITLEY 1931 : P.PERCIF. (Gobioid.) Gobiid.

Cassina COPE 1864 : errat. pro Kassina GIRARD 1853.

Cassinaëtus SCLATER 1922 : syn. de Spizaëtus VIEILLOT 1816.

Cassinia HARTLAUB 1860: Av. PASSERIF. (Acromyod.) Muscicapid.

Castanolimnas SHARPE 1893: Av. RALLIF. Rallid.

Castor LINNE 1758 : M.-RODENT. (Castoriomorph.) Castorid.

Casuarius LINNE 1758 : Av. STRUTHIONIF. Casuariid. (Casuariin.).

Catabasis EIGENMANN et NORRIS 1901: P.-CYPRINIF. (Characoid.) Characid.

Catablemella EIGENMANN 1891: P.-CLUPEIF. (Myctophoid.) Myctophiid.

Catadyptes MATHEWS 1924: Av. SPHENISCIF. Spheniscid.

Catalufa SNYDER 1911: P.-PERCIF. (Percoid.) Pempherid.

Catamblyrhynchus de LAFRESNAYE 1842: Av. PASSERIF. (Acromyod.) Fringillid. (Pitylin.).

Catamenia BONAPARTE $1850: A v$. PASSERIF. (Acromyod.) Fringillid.

Cataphororhynchus BIBRON 1855 : P. - TETRAODONTIF. (Tetraodontoid.) Lagocephalid.

Cataphractops FOWLER 1915 : P.-CYPRINIF. (Siluroid.) Callichthyid.

Catarrhactes HOMBRON et JACQUINOT : syn. de Pucherhamphus MATHEWS 1935.

Catathyridium CHABANAUD 1928 : P.-PLEURONECTIF. (Soleoid.) Soleid.

Cateorus KOLENATI 1856 : syn. de Eptesicus RAFINESQUE 1820.

Catharacta BRUNNICH 1764: Av. LARIF. Stercorariid.

Catharina DUNKER 1882 : errat. pro Katarina GRAY 1847.

Catharista VIEILLOT 1816: Av. FALCONIF. Vulturid.

Catharma ELLIOT 1876 : Av. APODIF. Trochilid.
Catharrhactes BRISSON: syn. d'Eudyptes VIEILLOT 1816.

Cathartes ILLIGER 1811 : syn. de Coragyps LE MAOUT 1853.

Catharus BONAPARTE 1850: Av. PASSERIF. (Acromyod.) Turdid. (Turdin.).

Cathayemys LINDHOLM 1931 : R.TESTUDIN. (Thecoph.) Emydid.

Catherpes BAIRD 1858: Av. PASSERIF. (Acromyod.) Troglodytid.

Catheturus SWAINSON 1837: Av. PHASIANIF. Megapodid.

Cathorops JORDAN et GILBERT 1883 : P.-CYPRINIF. (Siluroid.) Bagrid.

Catla CUVIER et VALENCIENNES 1844 : P.-CYPRINIF. (Cyprinoid.) Cyprinid.

Catlocarpio BOULENGER 1898: P.-CYPRINIF. (Cyprinoid.) Cyprinid.

Catochaenum CANTOR 1850 : P.PERCIF. (Percoid.) Leiognathid.

Catodiodon JACOB 1825: syn. d'Hyperoodon de LACEPEDE 1804.

Catodon LINNE 1761: syn. de Physeter LINNE 1758.

Catolynx SEVERTZOV 1858 : syn. de Felis LINNE 1758.

Catonotus AGASSIZ 1854 : P.-PERCIF. (Percoid.) Percid.

Catopra BLEEKER 1851 : P.-PERCIF. (Percoid.) Nandid.

Catoprion MULLER et TROSCHEL 1844: P.-CYPRINIF. (Characoid.) Characid.

Catoptrophorus BONAPARTE 1827: Av. CHARADRIIF. Charadriid. (Scolopacin.).

Catopuma SEVERTZOV 1858 : syn. de Felis LINNE 1758.

Catostomus LESUEUR 1817 : P.CYPRINIF. (Cyprinoid.) Catostomid.

Catulus SMITH 1838 : syn. de Scyliorhinus de BLAINVILLE 1816.

Catus FITZINGER 1855: syn. de Felis LINNE 1758. 
Catreus CABANIS 1851: Av. PHASIANIF. Phasianid. (Phasianin.).

Caudania ROULE 1935: P.-CLUPEIF. (Alepocephaloid.) Alepocephalid.

Caularchus GILL 1862 : P.-PERCIF. (Gobiesoxoid.) Gobiesocid.

Caulichthys OGILBY 1898 : P.-PERCIF. (Gobioid.) Eleotrid.

Caulolatilus GILL 1862 : P.-PERCIF. (Percoid.) Latilid.

Caulolepis GILL 1883: P.-BERYCIF. Berycid.

Caulophryne GOODE et BEAN 1896 : P.-LOPHIIF. (Ceratoid.) Ceratiid.

Caulopsetta GILL 1893 : syn. d'Arnoglossus BLEEKER 1862.

Caulopus GILL 1862 : P.-CLUPEIF. (Alepisauroid.) Alepisaurid.

Cauque EIGENMANN 1928 : P.-MUGILIF. Atherinid.

Causus WAGLER 1830 : R.-SQUAMAT. (Serpent.) Viperid.

Cavia PALLAS 1766 : M.-RODENT. (Caviomorph.) Caviid. (Caviin.).

Caviella OSGOOD $1915: s . / g$. de Microcavia GERVAIS et AMEGHINO 1880.

Cavivola LUTZ $1930: s . / g$. de Leptodactylus FITZINGER 1826.

Cayennia SAUVAGE 1880 : syn. de Gobioides de LACEPEDE 1800.

Cayleyna IREDALE 1930 : Av. PASSERIF. (Acromyod.) Ploceid. (Estrildin.).

Cebidichthys AYRES 1854 : P.-PERCIF. (Blennioid.) Blenniid.

Cebus ERXLEBEN 1777: M.-PRIMAT. (Simioid.) Cebid. (Cebin.).

Cecilia MATHEWS 1918 : syn. de Ceciliella STRAND 1928.

Ceciliella STRAND 1928: Av. CORACIADIF. Alcedinid.

Cecractana STRAND 1928: Av. CUCULLIF. Cucullid.

Cecractes ROBERTS 1922 : syn. de Cecractana STRAND 1928.

Celaenomys THOMAS 1898: M.-
RODENT. (Myomorph.) Murid. (Hydromyin.).

Celebesia RILEY 1918 : syn. de Celebesica STRAND 1928.

Celebesica STRAND 1928: Av. PASSERIF. (Acromyod.) Campephagid.

Celema GOODE et BEAN 1896 : P.PERCIF. (Ophidioid.) Brotulid.

Celestus GRAY 1838 : R.-SQUAMAT. (Saur.) Anguid. (serait syn. de Diploglossus WIEGMANN 1834).

Celeopicus MALHERBE 1849 : Av. PICIF. Picid. (Picin.).

Celeus BOIE 1831: Av. PICIF. Picid. (Picin.).

Cemas GLOGLER 1841: syn. de Rupicapra de BLAINVILLE 1816.

Cemophora COPE 1860 : R.-SQUAMAT. (Serpent.) Colubrid.

Cenisophius BONAPARTE 1845: P. CYPRINIF. (Cyprinoid.) Cyprinid.

Centetes ILLIGER 1811: syn. de Tenrec de LACEPEDE 1799.

Centracanthus RAFINESQUE 1810: P.-PERCIF. (Percoid.) Maenid.

Centracion GRAY 1831 : errat. pro Cestracion WALBAUM 1792.

Centranodon de LACEPEDE 1803 : P.-PERCIF. (Scorpaenoid.) Platycephalid.

Centrarchus CUVIER 1829: P.PERCIF. (Percoid.) Centrarchid.

Centratherina REGAN 1914: P.MUGILIF. Atherinid.

Centridermichthys RICHARDSON 1841: P.-PERCIF. (Cottoid.) Cottid.

Centrina CUVIER 1817 : syn. d'Oxynotus RAFINESQUE 1810.

Centriscops GILL 1862 : P.-AULOSTOMF. Macrorhamphosid.

Centriscus LINNE 1758 : syn. d'Amphisile KLEIN 1744.

Centrites CABANIS 1847 : Av. PASSERIF. (Mesomyod.) Tyrannid. (Fluvicolin.).

Centristhmus GARMAN 1899 : P.PERCIF. (Percoid.) Serranid. 
Centroberyx GILL 1862 : P.-BERYCIF. Berycid.

Centrocercus SWAINSON 1832: Av. PHASIANIF. Tetraonid.

Centrocetus REGAN et TREWAVAS 1932: P. LOPHIIF. (Ceratoid.) Ceratiid.

Centrochir AGASSIZ 1829 : P.-CYPRINIF. (Siluroid.) Doradid.

Centrochromis NORMAN 1922 : P.PERCIF. (Labroid.) Pomacentrid.

Centrococcyx CABANIS et HEINE 1863: Av. CUCULLIF. Cucullid.

Centrodoras EIGENMANN 1925: P.-CYPRINIF. (Siluroid.) Doradid.

Centrodraco REGAN 1913: P.-PERCIF. (Callionymoid.) Draconettid.

Centrogaster HOUTTUYN 1782: syn. de Siganus FORSKAL 1775 .

Centrogenys CUVIER et VALENCIENNES nec RICHARDSON 1842 : P.-PERCIF. (Percoid.) Serranid.

Centrogobius BLEEKER 1874 : P.PERCIF. (Gobioid.) Gobiid.

Centrolabrus GUNTHER 1861 : P.PERCIF. (Labroid.) Labrid.

Centrolene J. de La ESPADA 1872: Amph. ANOUR. Leptodactylid.

Centrolenella NOBLE 1920 : Amph. ANOUR. Hylid

Centrolophus de LACEPEDE 1802: P.-PERCIF. (Stromateoid.) Stromateid.

Centromochlus KNER 1858: P.CYPRINIF. (Siluroid.) Bagrid.

Centronotus SCHNEIDER 1801: syn. de Pholis GRONOV 1760.

Centronycteris GRAY 1838: s./g. de Saccopteryx ILLIGER 1811.

Centropelma SCLATER et SALVIN 1869: Av. COLYMBIF. Podicipid.

Centrophanes CABANIS 1845 : syn. de Centriles CABANIS 1847.

Centropholis HILGENDORF 1878 : P.-CORYPHAENIF. Bramid.

Centrophoroides DAVIS 1887 : P.SQUALIF. (Squalioid.) Squalid.
Centrophorus MULLER et HENLE 1837: P.SQUALIF. (Squalioid.) Squalid.

Centrophorus KNER 1859 : P.-CYPRINIF. (Characoid.) Characid.

Centrophryne REGAN et TREWAVAS 1932 : P.-LOPHIIF. (Ceratoid.) Ceratiid.

Centropodus de LACEPEDE 1802 : P.-PERCIF. (Percoid.) Toxotid.

Centropogon GUNTHER 1860 : P.PERCIF. (Scorpaenoid.) Scorpaenid.

Centropomus de LACEPEDE 1802: P.-PERCIF. (Percoid.) Serranid.

Centropristis CUVIER 1829: P.PERCIF. (Percoid.) Serranid.

Centropus ILLIGER 1811: Av. CUCULLIF. Cucullid.

Centropyge KAUP 1860 : P.-PERCIF. (Percoid.) Chaetodontid.

Centropyx CUVIER 1829: vide KENTROPYX SPIX 1825

Centroscyllium MULLER et HEN$L E$ 1841: P.-SQUALIF. (Squalioid.) Squalid.

Centroscymnus BOCAGE et CAPELLO 1864: P. -SQUALIF. (Squalioid.) Squalid.

Centroselachus GARMAN 1913 : P.SQUALIF. (Squalioid.) Squalid.

Centrurophis KAUP 1856: P.-ANGUILLIF. (Congroid.) Ophichthyid.

Centurio GRAY 1842: M.-CHIROPT. (Microchiropt.) Phyllostomatid. (Stenodermin.).

Centuriosus GRAY 1862 : syn. de Sus LINNE 1758.

Centurus SWAINSON 1837: Av. PICIF. Picid. (Picin.).

Ceophloeus : errat, pro Coephloeus CABANIS et HEINE 1862.

Cephacandia RAFINESQUE 1815 : syn. de Dactylopterus de LACEPEDE 1802.

Cephalacanthus de LACEPEDE 1802 : syn. de Dactylopterus de LACEPEDE 1802.

Cephalepis RAFINESQUE 1810 : P.-LAMPRIDIF. Trachypterid.

Cephaleutherus RAFINESQUE 1810 : P.-RAJIF. (Rajoid.) Rajid. 
Cephalinus GUNTHER 1854: P.PERCIF. (Scorpaenoid.) Congiopid.

Cephallepis LODDIGES 1830:Av. APODIF. Trochilid.

Cephalocassis BLEEKER 1858 : P.CYPRINIF. (Siluroid), Bagrid.

Cephalocottus GRATZIANOV 1907: P.-PERCIF. (Cottoid.) Cottid.

Cephalogobius BLEEKER 1874: P.-PERCIF. (Gobioid.) Gobiid.

Cephalokompsus : P. - CYPRINIF. (Cyprinoid.) Cyprinid.

Cephalolepis DUMERIL et BIBRON $1844: R .-S Q U A M A T$. (Serpent.) Typhlopid.

Cephalopholis SCHNEIDER 1801 : P.-PERCIF. (Percoid.) Serranid.

Cephalophus SMITH 1827 : M.-PARAX. (Solenodont.) Bovid. (Cephalophin.).

Cephalopsis RAFINESQUE 1815 : P.-CYPRINIF. (Cyprinoid.) Cyprinid.

Cephaloptera FLEMMING 1822 : syn. de Dicerobatis de BLAINVILLE 1816.

Cephalopterus RISSO 1810 : syn. de Mobula RAFINESQUE 1810.

Cephalopterus GEOFFROY-ST HILAIRE 1809: Av. PASSERIF. (Mesomyod.) Cotingid.

Cephalopyrus BONAPARTE 1854: Av. PASSERIF. (Acromyod.) Parid.

Cephalorhynchus GRAY 1846 : M.CETAC. (Odontocet.) Delphinid.

Cephaloscyllium GILL 1861: P.GALEIF. (Carcharhinoid.) Scyliorhinid.

Cephalosilurus HASEMAN 1911 : P.-CYPRINIF. (Siluroid.) Bagrid.

Cephalosimus CHABANAUD 1916 : s./g. de Simocephalus GUNTHER 1858.

Cephalotes GEOFFROY-ST-HILAIRE 1810 : syn. de Dobsonia PALMER 1898.

Cephalurus BIGELOW et SCHOEDER 1941 : P.-GALEIF. (Carcharhinoid.) Scyliorhinid.
Cephalus de LACEPEDE 1800 : P.CYPRINIF. (Cyprinoid.) Cyprinid.

Cephimmus RAFINESQUE 1815: P.-PERCIF. (Percoid.) Percid.

Cephus SWAINSON 1838: P.-GADIF. Gadid.

Cepola LINNE 1764: P.-PERCIF. (Percoid.) Cepolid.

Cepolophis KAUP 1856 : P.-PERCIF. (Percoid.) Cepolid.

Cenohus PALLAS 1769: syn. de Uria MOEHRING 1758.

Ceramodactylus BLANFORD 1874 : R.-SQUAMAT. (Saur.) Geckonid.

Cerasophila BINGHAM 1900: Av. PASSERIF. (Acromyod.) Pycnonotid.

Cerastes WAGLER 1830 : R.-SQUAMAT. (Serpent.) Viperid.

Ceratacanthus GILL 1861 : P.-PERCIF. (Balistoid.) Balistid. (ou Monacanthid.).

Ceratias KROYER 1845: P.-LOPHIIF. (Ceratoid.) Ceratiid.

Ceratichthys BAIRD et GIRARD 1854: P.-CYPRINIF. (Cyprinoid.) Cyprinid.

Ceratobatis BOULENGER 1897: P.-RAJIF. (Dasyatoid.) Mobulid.

Ceratobatrachus BOULENGER 1887: Amph. ANOUR. Hylid.

Ceratobranchia EI GE NMANN, HENN et WILSON 1914: P.CYPRINIF. (Characoid.) Characid.

Ceratocaulophryne ROULE et ANGEL 1932 : P.-LOPHIIF. (Ceratoid.) Ceratiid.

Ceratocheilus RIBEIRO 1918 : P.CYPRINIF. (Siluroid.) Doradid.

Ceratoglanis MYERS 1938 : P.-CYPRINIF. (Siluroid.) Silurid.

Ceratogymna BONAPARTE 1854 : Av. CORACIADIF. Bucerotid.

Ceratohyla (J. de La ESPADA 1870) BOULENGER 1882: Amph. ANOUR. Leptodactylid.

Ceratophora GRAY 1835: R.-SQUAMAT. (Saur.) Agamid.

Ceratophrys WIED 1824: Amph. ANOUR. Leptodactylid. 
Ceratopipra BONAPARTE 1854: Av. PASSERIF. (Mesomyod.) Piprid.

Ceratoptera MULLER et HENLE 1837: syn. de Manta BANCROFT 1829.

Ceratorhinus GRAY 1867: M.-PERISSODACT. (Ceratomorph.) Rhinocerotid. (Dicerorhin.).

Ceratoscopelus GUNTHER 1864 : syn. de Lampanyctus BONAPARTE 1840.

Ceratostethus MYERS 1937: P.CYPRINODONTIF. Phallostethid.

Ceratotriccus CABANIS 1874: Av. PASSERIF. (Mesomyod.) Tyrannid. (Platyrhinchin.).

Cerberus CUVIER 1829 : R.-SQUAMAT. (Serpent.) Homalopsid.

Cercaërtus BURMEISTER 1837 : M.-MARSUP. Phalangerid. (Phalangerin.).

Cerchneipicus BONAPARTE 1854 : Av. PICIF. Picid. (Picin.).

Cerchneis BUIE 1826 : Av. FALCONIF. Falconid. (Falconin.).

Cercocebus GEOFFROY-ST-HILAIRE 1812: M.-PRIMAT. (Simioid.) Cercopithecid. (Cercopithecin.).

Cercococcyx CABANIS 1882: Av. CUCULLIF. Cucullid.

Cercolabes BRANDT 1835 : syn. de Coendou de LACEPEDE 1799.

Cercoleptes ILLIGER 1811 : syn. de Potos GEOFFROY-ST-HILAIRE et CUVIER 1795.

Cercomacra SCLATER 1858: Av. PASSERIF. (Mesomyod.) Formicariid. (Formicariin.).

Cercomela BONAPARTE 1856 : Av. PASSERIF. (Acromyod.) Turdid.

Cercomitus : errat. pro Ceromitus WEBER 1913.

Cercomys CUVIER 1829 : M.-RODENT. (Caviomorph.) Echimyid. (Echimyin.).

Cercopithecus BRUNNICH 1772: M.-PRIMAT. (Simioid.) Cercopithecid. (Cercopithecin.).

Cercopithecus ERXLEBEN 1777: syn. de Leontocebus WAGNER 1839.
Cerconsis BILLBERG 1828: Av. RALLIF. Rallid.

Cercopteropus BURNETT 1829 : syn. de Rousettus GRAY 1821.

Cercorax: errat. pro Corcorax LESSON 1831.

Cercorhinca : errat. pro Cerorhyncha BONAPARTE 1828.

Cercosaura WAGLER 1830: R.SQUAMAT. (Saur.) Tejid.

Cercotrichas BOIE 1831 : Av. PASSERIF. (Acromyod.) Turdid.

Cerdale JORDAN et GILBERT 1882: syn. de Microdesmus GUNTHER 1864.

Cerdocyon SMITH 1839: M.-FISSIP. Canid. (Canin.).

Cereopsis LATHAM 1801: Av. ANATIF. Anatid. (Anserin.).

Cerivoula BLANFORD 1891: syn. de Kerivoula GRAY 1842.

Cerna BONAPARTE 1846 : P.-PERCIF. (Percoid.) Serranid.

Ceromitus WEBER 1913: P.-ANGUILLIF. (Nemichthyoid.) Nemichthyid.

Cerorhyncha BONAPARTE 1828 : Av. ALCIF. Alcid. (Fraterculin.).

Certhia LINNE 1758: Av. PASSERIF. (Acromyod.) Certhiid.

Certhialauda BURMEISTER 1850 : emed. pro Certhilauda SWAINSON 1827.

Certhiaxis LESSON 1847 : Av. PASSERIF. (Mesomyod.) Furnariid.

Certhidea GOULD 1837 : Av. PASSERIF. (Acromyod.) Fringillid. (Geospizin.).

Certhilauda SWAINSON 1827 : Av. PASSERIF. (Acromyod.) Alaudid.

Certhiola SUNDEVALL 1836 : Av. PASSERIF. (Acromyod.) Coerebid.

Certhiparus de LAFRESNAYE 1842: Av. PASSERIF. (Acromyod.) Parid. ou Campephagid.

Certhius OLPHE-GALLIARD 1888: errat. pro Certhia LINNE 1758.

Cervaria GRAY 1867 : syn. de $\mathrm{Fe}$ lis LINNE 1758.

Cervicapra SPARRMAN 1780 : syn. d'Antilope PALLAS 1766. 
Cervinipitta ELLIOT 1870: Av. PASSERIF. (Mesomyod.) Pittid.

Cervulus de BLAINVILLE 1816 : syn. de Muntiacus RAFINESQUE 1815.

Cervus LINNE 1758: M.-PARAX. (Solenodont.) Cervid.

Ceryle BOIE 1827: Av. CORACIADIF. Alcedinid.

Cerylealcyon: Av. CORACIADIF. Alcedinid.

Cestracion WALBAUM 1792 : syn. d'Heterodontus de BLAINVILLE 1816.

Cestraeus (MC CLELLAND 1841) AGASSIZ 1845: P.-GALEIF. (Carcharhinoid.) Sphyrnid.

Cestraeus CUVIER et VALENCIENNES 1836 : P.-MUGILIF. Mugilid.

Cestreus WALBAUM 1792 : P.-PERCIF. (Percoid.) Sciaenid.

Cestrorhinus de BLAINVILLE 1816 : P.-GALEIF. (Carcharhinoid.) Sphyrnid.

Cetengraulis GUNTHER 1868 : P.CLUPEIF. (Clupeoid.) Engraulid.

Cetodiodon JACOB 1825: syn. d'Hyperoodon de LACEPEDE 1804.

Cetomimoides KOEFOED 1955 : P.STEPHANOBERYCIF. Cetomimid.

Cetomimus GOODE et BEAN 1894: P.-STEPHANOBERYCIF. Cetomimid.

Cetonurus GUNTHER 1887: P.GADIF. Macrurid.

Cetopsis AGASSIZ 1829 : P.-CYPRINIF. (Siluroid.) Bagrid.

Cetopsorhamdia EIGENMANN 1916 : P.-CYPRINIF. (Siluroid.) Bagrid.

Cetorhinus de BLAINVILLE 1816 : P.-GALEIF. (Isuroid.) Cetorhinid.

Cetostoma ZUGMAYER 1914: P.STEPHANOBERYCIF. Cetomimid.

Cettia BONAPARTE 1834: Av. PASSERIF. (Acromyod.) Sylviid.

Cetupa LYDEKKER 1891 : syn. de Keputa LESSON 1830.
Ceuthmochares CABANIS et HEINE 1863: Av. CUCULLIF. Cucullid.

Ceyx de LACEPEDE 1799 : Av. CORACIADIF, Alcedinid.

Chabanaudetta WHITLEY 1931: P.-PLEURONECTIF. (Soleoid.) Soleid.

Chabanaudia de WITTE et LAURENT 1942 : R.-SQUAMAT. (Saur.) Scincid.

Chaca CUVIER et VALENCIENNES 1839 : P.-CYPRINIF. (Siluroid.) Plotosid.

Chacomys OSGOOD $1946: s . / g$. de Ctenomys de BLAINVILLE 1826.

Chaenichthys RICHARDSON 1844 : P.-PERCIF. (Trachinoid.) Trachinid.

Chaenobrythus GILL 1864 : P.PERCIF. (Percoid.) Centrarchid.

Chaenocephalus $R E G A N 1913$ : P.-PERCIF. (Cottoid.) Cottid.

Chaenocetus ESCHRICHT 1846 : syn. d'Hyperoodon de LACEPEDE 1804.

Chaenodelphinus ESCHRICHT 1843 : syn. d'Hyperoodon de LACEPEDE 1804.

Chaenogaleus GILL 1861: P.-GALEIF. (Carcharhinoid.) Scyliorhinid.

Chaenogobius GILL 1858 : P.-PERCIF. (Gobioid.) Gobiid.

Chaenomugil GILL 1863: P.-MUGILIF. Mugilid.

Chaenophryne REGAN 1925: P.LOPHIIF. (Ceratoid.) Oneiro-

Chaenopsis GILL 1865: P.-PERCIF. (Blennioid.) Clinid.

Chaenothorax COPE 1878 : P.-CYPRINIF. (Siluroid.) Callichthyid.

Chaenotropus : P.-CYPRINIF. (Characold.) Characid.

Chaerophon DOBSON $1874: s . / g$. de Tadarida RAFINESQUE 1814.

Chaeropithecus GERVAIS 1839 : syn. de Papio MULLER 1773.

Chaerops : errat. pro Choerops RUPPELL 1852. 
Chaeropsodes GILCHRIST et THOMPSON 1909 : P.-PERCIF. (Labroid.) Labrid.

Chaeropus OGILBY 1838 : M.-MARSUP. Peramelid.

Chaetichthys GISTEL 1848: P.PERCIF. (Trachinoid.) Trachinia.

Chaetobranchopsis STEINDACHNER 10ı́ : P.-PERCIF. (Percoid.) Cichlid.

Chaetobranchus HAECKEL 1840 : P.-PERCIF. (Percoid.) Cichlid.

Chaetocercus GRAY 1855: Av. APODIF. Trochilid.

Chaetocercus KREFFT 1867 : syn. de Dasycercus PETERS 1875.

Chaetodipterus de LACEPEDE 1802: P.-PERCIF. (Percoid.) Ephippuid.

Chaetodipus MERRIAM 1889 : s./g. de Perognathus WIED 1839.

Chaetodon LINNE 1758: P.-PERCIF. (Percoid.) Chaetodontid.

Chaetodontoplus BLEEKER 1876 : P.-PERCIF. (Percoid.) Chaetodontid.

Chaetodontops BLEEKER 1876 : P.-PERCIF. (Percoid.) Chaetodontid.

Chaetoessus $S$ W A I N SON 1838 : errat. pro Chatoessus CUVIER 1829.

Chaetolabrus SWAINSON 1839 : P.-PERCIF. (Labroid.) Labrid.

Chaetomus Mc CLELLAND 1843 : P.-CLUPEIF. (Cluepoid.) Engraulid.

Chaetomys GRA Y 1843: M.-RODENT. (Caviomorph.) Erethizontid. (Chaetomyin.).

Chaetonhractus FITZINGER 1871 : M.-EDENT. (Xenarthr.) Dasypodid. (Euphractin.).

Chaetops SWAINSON 1832: Av. PASSERIF. (Acromyod.) Timaliid.

Chaetopterus TEMMINCK et SCHLEGEL 1844: P.-PERCIF. (Percoid.) Lutjanid.

Chaetoptila SCLATER 1871: Av. PASSERIF. (Acromyod.) Meliphagid.

Chaetorhynchus MEYER 1874: Av.
PASSERIF. (Acromyod.) Dicrurid.

Chaetornis GRAY 1848: Av. PASSERIF. (Acromyod.) Timaliid.

Chzetostoma TSCHUDI 1846 : P.-CYPRINIF. (Siluroid.) Loricariid.

Chaetostomus HAECKEL 1870 : P.PERCIF. (Percoid.) Cichlid.

Chaetura STEPHENS 1826: Av. APODIF. Apodid.

Chaeturichthys RICHARDSON 1844: P.-PERCIF. (Gobioid.) Gobiid.

Chaetusia AGASSIZ 1846: emend. pro Chettusia BONAPARTE 1838.

Chagunius SMITH 1938 : P.-CYPRINIF. (Cyprinoid.) Cyprinid.

Chaibassia THEOBALD 1876: R.TESTUDIN. (Thecoph.) Testudinid.

Chalaroderma NORMAN 1943 : P.-PERCIF. (Blennioid.) Blenniid.

Chalarodon P E T E R S 1854 : R.SQUAMAT. (Saur.) Iguanid.

Chalcacomus : errat. pro Chalcocomus RILEY 1934.

Chalcalburnus BERG 1932 : P.-CYPRINIF. (Cyprinoid.) Cyprinid.

Chalceus CUVIER 1818 : P.-CYPRINIF. (Characoid.) Characid.

Chalcides LAURENTI 1768 : R.-SQUAMAT. (Saur.) Scincid.

Chalcidoseps BOULENGER 1887 : R.-SQUAMAT. (Saur.) Scincid.

Chalcinopelecus HOLMBERG 1892: P.-CYPRINIF. (Characoid.) Characid.

Chalcinopsis KNER 1863 : P.-CYPRINIF. (Characoid.) Characid.

Chalcinus CUVIER et VALENCIENNES 1849 : P.-CYPRINIF. (Characoid.) Characid.

Chalcites LESSON 1830: Av. CUCULLIF. Cucullid.

Chalcococcyx CABANIS et HEINE 1863: Av. CULULLIF. Cucullid.

Chalcocomus RILE Y 1934: Av. PHASIANIF. Phasianid. (Phasianin.). 
Chalcomitra REICHENBACH 1853 : Av. PASSERIF. (Acromyod.) Nectariniid.

Chalcomys THOMAS 1916: syn. d'Akodon MERREM 1833.

Chalcoparia CABANIS 1851: Av. PASSERIF. (Acromyod.) Nectarinitd.

Chalconelia BONAPARTE 1854: Av. COLUMBIF. Columbid. (Columbin.).

Chalcopelix : errat, pro Chalcopelia BONAPARTE 1854.

Chalcophaps GOULD 1843: Av. COLUMBIF. Columbid. (Columbin.).

Chalcophasis ELLIOT 1871: Av. PHASIANIF. Phasianid. (Phasianin.).

Chalcopsar SHARPE 1890: Av. PASSERIF. (Acromyod.) Sturnid.

Chalcopsitta BONAPARTE 1849 : Av. PSITTACIF. Psittacid.

Chalcopsittacus SUNDEVALL 1872: errat. pro Chalcopsitta B. 1849.

Chalcostetha CABANIS 1851: Av. PASSERIF. (Acromyod.) Nectariniid.

Chalcostigma REICHENBACH 1853: Av. APODIF. Trochilid.

Chalcurus BONAPARTE 1854:Av. PHASIANIF. Phasianid. (Phasianin.).

Chalictes: errat. pro Chalcites LESSON 1830.

Chalidichthys : P.-PERCIF. (Percoid.) Serranid.

Chalinobotus PETERS : $M$. CHIROPT. (Microchiropt.) Vespertilionid. (Vespertilionin.).

Chalinura GOODE et BEAN 1882: syn. de Fuyangia WHITLEY 1931.

Chalinurus GUNTHER 1887: P.-GADIF. Macrurid.

Chalixodytes $S C H U L T Z 1943$ : P.-PERCIF. (Trachinoid.) Pinguipedid.

Chalybura REICHENBACH 1854: syn. d'Hypuroptila GOULD 1854.

Chalygama MATHEWS 1925: Av. PASSERIF. (Acromyod.) Paradiseid. (Paradisein.).
Chamaea GAMBEL 1847 : Av. PASSERIF. (Acromyod.) Chamaeid.

Chamaeleolis DUMERIL et BIBRON 1837: R. - SQUAMAT. (Saur.) Iguanid.

Chamaeleon GRONOV 1763: R.SQUAMAT. (Saur.) Chamaeleonid.

Chamaeleontuceps: P.-PERCIF. (Labroid.) Pomacentrid.

Chamaelinorops SCHMIDT 1919 : R.-SQUAMAT. (Saur.) Iguanid.

Chamaeospiza SCLATER 1858 : Av. PASSERIF. (Acromyod.) Fringillid.

Chamaeopelia (SWAINSON 1827) SCLATER 1864: syn. de Columbigallina OKEN 1817.

Chamaepetes WAGLER 1832: Av. PHASIANIF. Cracid.

Chamaesaura SCHNEIDER 1801: R.-SQUAMAT. (Saur.) Zonurid.

Chamaethlvpis RIDGWAY 1887: Av. PASSERIF. (Acromyod.) Parulid.

Chamaetortus GUNTHER 1864: R.-SQUAMAT. (Serpent.) Boigid.

Chamaeza VIGORS 1825 : Av. PASSERIF. (Mesomyod.) Formicariid. (Grallarin.).

Chamaezosa CABANIS 1847 : errat. pro Chamaeza VIGORS 1825.

Chamaigenes EIGENMANN 1912 : P.-CYPRINIF. (Siluroid.) Bunocephalid.

Champse MERREM 1820: syn. de Crocodilus LINNE 1758.

Champsoborus BOULENGER 1909: P.-CYPRINIF. (Characoid.) Characid.

Champsocephalus GILL 1861 : P.PERCIF. (Trachinoid.) Nototheniid.

Champsochromis BOULENGER 1916: P.-PERCIF. (Percoid.) Cichlid.

Champsodon GUNTHER 1867 : P.-PERCIF. (Chiasmodontoid.) Chiasmodontid.

Chanda HAMILTON-BUCHANAN 1822 : P.-PERCIF. (Percoid.) Serranid.

Channa GRONOV 1763 : P.-PERCIF. 
(Ophiocephaloid.) Ophiocephalid.

Channalabeo : P.-CYPRINIF. (Siluroid.) Clariid.

Channallabes G U N TH E R 1873 : P.-CYPRINIF. (Siluroid.) Clariid.

Channichthys : errat. pro Chaenichthys RICHARDSON 1844.

Channomuraena RICHARDSON 1844 : P.-ANGUILLIF. (Anguilloid.) Muraenid.

Chanodichthys BLEEKER 1860 : P.-CYPRINIF. (Cyprinoid.) Cyprinid.

Chanos de LACEPEDE 1803 : P.CLUPEIF. (Chanoid.) Chanid.

Chapalichthys MEEK 1902 : P.-CYPRINODONTIF. Goodeid.

Chaparrudo de BUEN 1931 : P.PERCIF. (Gobioid.) Gobiid.

Chaperina $M O C Q U A R D 1892$ : Amph. ANOUR. Microhylid.

Chapinortyx ROBERTS 1928: Av. PHASIANIF. Phasianid. (Phasianin.).

Chapmania RIBEIRO 1920: syn. de Chapmaniana STRAND 1928.

Chapmaniana STRAND 1928: Av. PSITTACIF. Psittacid.

Chaptia HODGSON'1837 : Av. PASSERIF. (Acromyod.) Dicrurid.

Characidium REINHARDT 1866 : P.-CYPRINIF. (Characoid.) Characid.

Characinus de LACEPEDE 1803 : syn. de Charax SCOPOLI 1777.

Characodon $G U N T H E R \quad 1866$ : P.-CYPRINODONTIF. Goodeid.

Charadrius LINNE 1758 : Av. CHARADRIIF. Charadriid. (Charadriin.).

Charalia de BUEN 1945: s./g. de Eslopsarum JORDAN et EVERMANN 1897.

Charantillas : errat. pro Charitilla OBERHOLSER 1905.

Charanx SWAINSON 1839 : syn. de Caranx de LACEPEDE 1802.

Charax SCOPOLI 1777 : P.-CYPRINIF. (Characoid.) Characid.

Charaxodon FERNANDEZ-YEPEZ 1947: P. - CYPRINIF. (Characoid.) Characid.
Charina GRAY 1849: R.-SQUAMAT. (Serpent.) Boid. (Erygin.).

Charisella FOWLER 1939 : P.-MUGILIF. Atherinid.

Charitillas OBERHOLSER 1905 : Av. PASSERIF. (Acromyod.) Pycnonotid.

Charitochelidon WOLTERS 1943 : s./g. d'Hirundo LINNE 1758.

Charitociris OBERHOLSER 1923: Av. PASSERIF. (Acromyod.) Dicaeid.

Charitonetta STEJNEGER 1886: Av. ANATIF. Anatid. (Anatin.).

Charitospiza OBERHOLSER 1905 : Av. PASSERIF. (Acromyod.) Fringillid.

Charmosyna WAGLER 1832: Av. PSITTACIF. Psittacid.

Charmosynopsis SALVADORI 1877: Av. PSITTACIF. Psittacid.

Charronia GRAY 1865: s./g. de Martes PINEL 1792.

Charybdia FACCIOLA 1885: P.-PLEURONECTIF. (Pleuronectoid.) Bothid.

Chascanopsetta ALCOCK 1847 : P.PLEURONECTIF. (Pleuronectoid.) Bothid.

Chasiempis CABANIS 1847: Av. PASSERIF. (Acromyod.) Muscicapid.

Chaemenchelys FOWLER 1944: P. - ANGUILLIF. (Anguilloid.) Muraenid.

Chasmias JORDAN et SNYDER 1901: syn. de Chasmichthys JORDAN 1903.

Chasmichthys JORDAN 1903 : P.PERCIF. (Gobioid.) Gobiid.

Chasmistes JORDAN 1878 : P.-CYPRINIF. (Cyprinoid.) Catostomid.

Chasmocenhalus EIGENMANN 1910 : P.-CYPRINIF. (Siluroid.) Bagrid.

Chesmocranus EIGENMANN 1912 : P.-CYPRINIF. (Siluroid.) Bagrid.

Chatoessus CUVIER 1829 : P.-CLUPEIF. (Clupeoid.) Dorosomid.

Chaudhuria ANNANDALE 1918 : P.-MASTCEMBELIF. Chaudhuriid. 
Chaulelasmus BONAPARTE 1838 : Av. ANATIF. Anatid. (Anatin.).

Chauliodus BLOCH et SCHNEIDER 1801: P.-CLUPEIF. (Stomiatoid.) Chauliodid.

Chauna ILLIGER 1811: Av. ANHI$M I F$. Anhimid.

Chaunax LOWE 1846: P.-LOPHIIF. (Antennarioid.) Chaunacid.

Chaunoproctus BONAPARTE 1850 : Av. PASSERIF. (Acromyod.) Fringillid. (Coccothraustin.).

Chaunornis GRAY 1841: Av. PICIF. Bucconid.

Chaus GRAY 1843 : syn. de Felis LINNE 1758.

Chedrus SWAINSON 1839 : P.-CYPRINIF. (Cyprinoid.) Cyprinid.

Cheilidonichthys : errat. pro Chelidonichthys KAUP 1872.

Cheilinoides $B L E E K E R$ 1851 : P.-PERCIF. (Labroid.) Labrid.

Cheilinus de LACEPEDE 1802: P.-PERCIF. (Labroid.) Labrid.

Cheilio : vide Chelio LACEPEDE 1802.

Cheiliopsis STEINDACHNER 1863 : P.-PERCIF. (Labroid.) Labrid.

Cheilobarbus SMITH 1841 : P.-CYPRINIF. (Cyprinoid.) Cyprinid.

Cheilobranchus RICHARDSON 1845 : P.-ALABIF. Alabid.

Cheilodactylus de LACEPEDE 1803: P.-PERCIF. (Percoid.) Cheilodactylid.

Cheilodipterops SCHULTZ 1940 : P.-PERCIF. (Percoid.) Serranid.

Cheilodipterus de LACEPEDE 1802: P.-PERCIF. (Percoid.) Serranid. (ou Cheilodipterid.).

Cheilolabrus A L L E Y N E et Mc LEAY 1877 : P.-PERCIF. (Labroid.) Labrid.

Cheilonemus STORER 1855: P.CYPRINIF. (Cyprinoid.) Cyprinid.

Cheilopogon LOWE 1841 : P.-BELONIF. (Exocoetoid.) Exocoetid.

Cheiloprion WEBER 1913: P.-PERCIF. (Labroid.) Pomacentrid.

Cheilotrema TSCHUDI 1846 : P.-PERCIF. (Percoid.) Sciaenid.
Cheimerius SMITH 1938 : P.-PERCIF. (Percoid.) Sparid.

Cheiragonus HERTZENSTEIN 1891: P.-PERCIF. (Cottoid.) Agonid.

Cheirocerus EIGENMANN 1917 : P.-CYPRINIF. (Siluroid.) Bagrid.

Cheirodontops SCHULTZ 1944: P.-CYPRINIF. (Characoid.) Characid.

Cheirodus PANDER 1858 : P.-CYPRINIF. (Siluroid.) Loricariid.

Cheirogaleus GEOFFROY ST-HILAIRE 181 L : M.-PRIMAT. (Lemuroid.) Lemurid. (Cheirogalein.).

Cheiromeles HORSFIELD 1824: M. - CHIROPT. (Microchiropt.) Molossid.

Cheiromys CUVIER 1800 : syn. de Daubentonia GEOFFROY ST-HILAIRE 1795.

Cheironebris FOWLER 1944: P.PERCIF. (Percoid.) Sciaenid.

Cheiroxenichthys FOWLER 1930 : s./g. de Xenichthys GILL 1863.

Chela HAMILTON - BUCHANAN 1822: P.-CYPRINIF. (Cyprinoid.) Cyprinid.

Chelaethiops BOULENGER 1899: P.-CYPRINIF. (Cyprinoid.) Cyprinid.

Chelemys THOMAS 1903 : syn. de Notiomys THOMAS 1890.

Chelemyscus THOMAS 1925: M.RODENT. (Myomorph.) Cricetid. (Hesperomyin.).

Chelictinia LESSON 1843: Av. FALCONIF. Falconid. (Accipitrin.).

Chelido BILLBERG 1828: errat. pro Chelidon BOIE 1822.

Chelidon BOIE 1822: Av. PASSERIF. (Acromyod.) Hirundinid.

Chelidonaria REICHENOW 1889 : syn. de Delichon HORSFIELD et MOORE 1854.

Chelidonichthys K A U P 1872 : P.PERCIF. (Scorpaenoid.) Triglid.

Chelidoperca BOULENGER 1894 : P.-PERCIF. (Percoid.) Serranid. 
Chelidoptera GOULD 1836: Av. PICIF. Bucconid.

Chelidorynx HODGSON 1844: Av. PASSERIF. (Acromyod.) Muscicapid.

Chelio de LACEPEDE 1802 : P.PERCIF. (Labroid.) Labrid.

Cheliones THOMAS $1910: s . / g$. de Meriones ILLIGER 1811.

Chelmon CUVIER et VALENCIENNES 1831: P.-PERCIF. (Percoid.) Chaetodontid.

Chelmonops $B L E E K E R \quad 1876$ : P.-PERCIF. (Percoid.) Chaetodontid.

Chelodina FITZINGER 1826 : R.TESTUDIN. (Thecoph.) Chelyid.

Chelon ARTEDI 1793: P.-MUGILIF. Mugilid.

Chelonia BROGNIART 1800 : R.TESTUDIN. (Thecoph.) Chelonutd.

Chelonodon $M U L L E R 1841$ : P.TETRAODONTIF. (Tetraodontoid.) Tetraodontid.

Chelonodontops SMITH 1958 : P. - TETRAODONTIF. (Tetraodontoid.) Tetraodontid.

Chelopus RAFINESQUE 1832 : R.TESTUDIN. (Thecoph.) Emydid.

Chelosauria : R.-SQUAMAT. (Saur.) Agamid.

Chelydra SCHWEIGGER 1812 : R.TESTUDIN. (Thecoph.) Chelydrid.

Chelys DUMERIL 1806 : R.-TESTUDIN. (Thecoph.) Chelyid.

Chen BOIE 1822: Av. ANATIF. Anatid. (Anserin.).

Chenalopex STEPHENS 1824 nec MOEHRING 1758: Av. ANA TIF. Anatid. (Anserin.).

Chendytes MILLER 1925: Av. ANATIF. Anatid. (Anserin.).

Chenia FOWLER 1958: syn. d'Othos CASTELNAU 1875.

Chenogaster LAHILLE 1904: P. - PERCIF. (Scombroid.) Scombrid.

Chenonetta B R A N D T 1836: Av. ANATIF. Anatid. (Anserin.).

Chenopis WAGLER 1832: Av. ANATIF, Anatid. (Anserin.).
Chenorhamphus OUSTALET 1878: Av. PASSERIF. (Acromyod.) Muscicapid.

Cheonda GIRARD 1856 : P.-CYPRINIF. (Cyprinoid.) Cyprinid.

Chera GRAY 1844 : Av. PASSERIF. (Acromyod.) Ploceid. (Viduin.).

Cheramoeca CABANIS 1851: Av. PASSERIF. (Acromyod.) Hirundinid.

Chersina GRAY 1831 : syn. de Goniochersus LINDHOLM 1929.

Chersine MERREM 1820 : s./g. de Testudo LINNE 1758.

Chersinella GRAY 1870: R.-TESTUDIN. (Thecoph.) Testudinid.

Chersodromus REINHARDT 1860 : R.-SQUAMAT. (Serpent.) Colubrid.

Chersomanes CABANIS 1851: Av. PASSERIF. (Acromyod.) Alaudid.

Chersophilus SHARPE 1890: Av. PASSERIF. (Acromyod.) Alaudid.

Chersydrus CUVIER 1817: R.SQUAMAT. (Serpent.) Colubrid.

Cherublemma TROTTER 1926: P.-PERCIF. (Ophidioid.) Brotulid.

Chettusia BONAPARTE 1838: Av. CHARADRIIF. Charadriid. (Charadriin.).

Chiasmocleis MEHLY 1904 : Amph. AvOUR. Microhylid.

Chiasmodon JOHNSON 1864 : P.-PERCIF. (Chiasmodontoid.) Cniasmodontid.

Chiasmichthys : errat. pro Chiasmichthys JORDAN 1903.

Chibia HODGSON 1837: Av. PASSERIF. (Acromyod.) Dicrurid.

Chicquera LESS $O N$ 1836: Av. FALCONIF. Falconid. (Falconin.).

Chilatherina REGAN 1914 : P.-MUGILIF. Atherinid.

Chilia SALVADORI 1908 : Av. PASSERIF. (Mesomyod.) Furnariid.

Chilias OGILBY 1910 : P.-PERCIF. (Trachinoid.) Pinguipedid.

Chilidonias : errat. pro Chlidonias RAFINESQUE 1822. 
Chilinus AGASSIZ 1846: emend. pro Cheilinus de LACEPEDE 1802.

Chilobranchus GUNTHER 1870 : errat. pro Cheilobranchus RICHARDSON 1845.

Chilochromis BOULENGER 1902 : P.-PERCIF. (Percoid) Cichlid.

Chiloconger MYERS 1941 : P.-ANGUILLIF. (Congroid.) Congrid.

Chilodactvlus A GASSIZ 1846 : emend. pro Cheilodactylus de LACEPEDE 1803.

Chilodinterus A GASSIZ 1846: emend. pro Cheilodipterus de LACEPEDE 1802.

Chilodus MULLER et TROSCHEL 1844 : P.-CYPRINIF. (Characoid.) Characid.

Chiloglanis PETERS 1868 : P.-CYPRINIF. (Siluroid.) Bagrid.

Chilogobio BERG 1915 : P.-CYPRINIF. (Cyprinoid.) Cyprinid.

Chilomeniscus COPE 1860: R.SQUAMAT. (Serpent.) Colubrid.

Chilomycterus KAUP 1846 nec BIBRON 1849 : P.TETRAODONTIF. (Teraodontoid.) Diodontid.

Chilomys THOMAS 1897: M.-RODENT. (Myomorph.) Cricetid. (Hesperomyin.).

Chilomyzon F O WL E R 1906 : P.CYPRINIF. (Characoid.) Characid.

Chilonatalus MILLER 1898: s./g. de Natalus GERVAIS 1855.

Chilonycteris GRAY 1839 : M.-CHIROPT. (Microchiropt.) Phyllostomatid. (Chilonycterin.).

Chilophylla MILLER 1911 : syn. de Coelops BLYTH 1848.

Chilopterus PHILIPPI 1858: Cyclost. PETROMYZONIF. Petromyzonid.

Chilorhinophis WERNER 1908: R.-SQUAMAT. (Serpent.) Boigid.

Chilorhinus LUTKEN 1852 : P.-ANGUILLIF. (Congroid.) Echelid.

Chiloscyllium MULLER et HENLE 1837 : P.-GALEIF. (Isuroid.) Orectolobid.

Chilotilapia BOULENGER 1908 : P.-PERCIF. (Percoid.) Cichlid.
Chilotus BAIRD 1857 : s./g. de Microtus SCHRANK 1798.

Chimaera LINNE 1758 : P.-HOLOCEPHAL. Chimaerid.

Chimarrichthys SAUVAGE 1874: P.-PERCIF. (Trachinoid.) Trachinid.

Chimarrogale ANDERSON 1877: M.-INSECTIV. Soricid. (Crocidurin.).

Chimarrhornis (HODGSON 1844) BEAVAN 1867: Av. PASSERIF. (Acromyod.) Timaliid.

Chimpanse VOIGT 1831: syn. de Pan OKEN 1816.

Chinchilla BENNETT 1829: M.RODENT. (Caviomorph.) Chinchillid.

Chinchillula THOMAS 1898: M.RODENT. (Myomorph.) Cricetid. (Hesperomyin.).

Chinemys SMITH 1931 : R.-TESTUDIN. (Thecoph.) Emydid.

Chioglossa BARBOZA du BOCAGE 1864: Amph. UROD. Salamandrid.

Chionactis COPE 1860 : R.-SQUAMAT. (Serpent.) Colubrid.

Chionarchus KIDDER et COUES 1876 : Av. CHARADRIIF. Chionid.

Chionis FORSTER 1788: Av. CHARADRIIF. Chionid.

Chionobates KAUP 1829 : syn. de Lepus LINNE 1758.

Chionochen REICHENBACH 1852 : Av. ANATIF. Anatid. (Anserin.).

Chionomys MILLER 1908: syn. de Microtus SCHRANK 1798.

Chionophilos PETENYI 1832: Av. PASSERIF. (Acromyod.) Ala.

Chionospina KAUP 1829 : Av. PASSERIF. (Acromyod.) Fringillid. (Fringillin.).

Chirindia BOULENGER 1907: R. SQUAMAT. (Saur.) Amphisbaenid.

Chirixalus $B O U L E N G E R 1893$ : Amph. ANOUR. Ranid.

Chirocentrodon GUNTHER 1868 : P.-CLUPEIF. (Clupeoid.) Chirocentrid.

Chirocentrus CUVIER 1817: P.- 
CLUPEIF. (Clupeoid.) Chirocentrid.

Chirocylla SCLATER et SALVIN 1876: Av. PASSERIF. (Mesomyod.) Cotingid.

Chirodactylus GILL 1862 : P.-PERCIF. (Percoid.) Cheilodactylid.

Chiroderma P E T E R S 1860 : M.CHIROPT. (Microchiropt.)

Plyllosiomatid. (Stenodermin.).

Chirodon GUNTHER 1864 : errat. pro Cheirodon GIRARD 1854.

Chirodorus Mc COY 1848 : P.-BELONIF. (Exocoetoid.) Hemirhamphid.

Chirogale GEOFFROY ST-HILAIRE 1829 : syn. de Cheirogaleus $G$. St-H. 1812.

Chirogalus GEOFFROY ST-HILAIRE 1812 : syn. de Cheirogaleus G. St-H. 1812.

Chiroleptes GUNTHER 1858 : Amph. ANOUR. Leptodactylid.

Chirolophis SWAINSON 1839 : P.PERCIF. (Blennioid.) Pholid.

Chirolophius REGAN 1903 : P.-LOPHIIF. (Lophioid.) Lophiid.

Chiromantis PETERS 1854: Amph. ANOUR. Rhacophorid.

Chiromys : vide Cheiromys $\mathrm{CU}$ VIER 1800.

Chiromyscus THOMAS 1925 : M.-RODENT. (Myomorph.) Murid. (Murin.).

Chironax ANDERSEN 1912 : syn. de Cynopterus CUVIER 1824.

Chironectes ILLIGER 1811: M.MARSUP. Didelphid.

Chironectes CUVIER 1817: syn. d'Antennarius (COMMERSON) de LACEPEDE 1798.

Chironemus CUVIER et VALENCIENNES 1829: P.-PERCIF. (Percoid.) Chironemid.

Chironius FITZINGER 1826: R.SQUAMAT. (Serpent.) Colubrid.

Chirophryne REGAN et TREWAVAS 1932 : P.-LOPHIIF. (Ceratoid.) Ceratiid.

Chiropodomys PETERS 1868 : M.RODENT. (Myomorph.) Murid. (Murin.).
Chiropotes LESSON 1840 : M.-PRIMAT. (Simioid.) Cebid. (Pithecin.).

Chiropsis GIRARD 1857 : P.-PERCIF. (Scorpaenoid.) Hexagrammid.

Chiropterotriton TAYLOR 1944: Amph. UROD. Plethodontid.

Chiroscaptor HEUDE 1897: syn. de Talpa LINNE 1766.

Chirostoma SWAINSON 1839 : P.MUGILIF. Atherinid.

Chirotes CUVIER 1817 : R.-SQUAMAT. (Saur.) Amphisbaenid.

Chiroxiphia CABANIS 1847: Av. PASSERIF. (Mesomyod.) Piprid.

Chiruromys THOMAS 1888: syn. de Pogonomys MILNE-EDWARDS 10.7.

Chirus PALLAS 1811 : P.-PERCIF. (Ophidioid.) Ophidiid.

Chitala FOWLER 1934: s./g. de Notopterus de LACEPEDE 1800.

Chitonotus LOCKINGTON 1882 : P.-PERCIF. (Cottoid.) Cottid.

Chitra GRAY 1844: R.-TESTUDIN. (Thecoph.) Trionychid.

Chlamydera GOULD 1837: Av. PASSERIF. (Acromyod.) Paradiseid. (Ptilonorhynchin.).

Chlamydes JENKINS 1904: P.-PERCIF. (Gobioid.) Gobiid.

Chlamydochen BONAPARTE 1854: Av. ANATIF. Anatid. (Anserin.).

Chlamydogobius WHITLEY 1930 : P.-PERCIF. (Gobioid.) Gobiid.

Chlamydophorus WAGLER 1830 : syn. de Chlamyphorus HARLAN 1825.

Chlamydosaurus GRAY 1826: R.SQUAMAT. (Saur.) Agamid.

Chlamydoselachus GARMAN 1884: P.-HEXANCHIF. Chlamydoselachid.

Chlamydotis LESSON 1839: Av. RALLIF. Otidid.

Chlamyphorus HARLAN 1825 : M.EDENT. (Xenarthr.) Dasypodid. (Chlamyphorin.).

Chlenasicus GIEBEL 1872 : errat. pro Chleuasicus BLYTH 1845. 
Chleuasicus BLYTH 1845: Av. PASSERIF. (Acromyod.) Timaliid.

Chlevastes JORDAN et SNYDER 1901: P.-ANGUILLIF. (Congroid.) Ophichthyid.

Chlidichthys SMITTH 1954: P.PERCIF. (Percoid.) Serranid. (ou Pseudochromid.).

Chlidonias RAFINESQUE 1822: Av. LARIF. Larid. (Sternin.).

Chloea JORDAN et SNYDER 1901 : syn. de Chloëichthys.

Chloëichthys WHITLEY 1940 : P.PERCIF. (Gobioid.) Gobiid.

Chloëphaga EYTON 1838: Av. A↔ATIF. Anatid. (Anserin.).

Chlopsis RAFINESQUE 1810 : P.ANGUILLIF. (Congroid.) Nettastomatid.

Chlorestes REICHENBACH 1854: Av. APODIF. Trochilid.

Chlorestrilda SHELLEY 1905: Av. PASSERIF. (Acromyod.) Fringillid. (Estrildin.).

Chlorichthys SWAINSON 1839 : P.PERCIF. (Labroid.) Labrid.

Chloridops WILSON 1888: Av. PASSERIF. (Acromyod.) Drepanidid.

Chloris CUVIER 1800 : Av. PASSERIF. (Acromyod.) Fringillid. (Fringillin.).

Chlorisitta MATHEWS 1935: Av. PASSERIF. (Acromyod.) Fringillid. (Fringillin.).

Chloristes MULSANT et VERREAUX 1866 : errat. pro Chlorestes REICHENBACH 1854.

Chloroceryle KAUP 1848 : Av. CORACIADIF. Alcedinid.

Chlorocharis SHARPE 1888: Av. PASSERIF. (Acromyod.) Zosteropid.

Chlorochrysa BONAPARTE 1851: Av. PASSERIF. (Acromyod.) Tanagrid.

Chlorocichla SHARPE 1881: Av. PASSERIF. (Acromyod.) Pycnonotid.

Chlorodrepanis ROTSCHILD 1900: Av. PASSERIF. (Acromyod.) Drepanidid.

Chlorolampis CABANIS et HEINE 1860 : Av. APODIF. Trochilid.
Chloromonarcha MATHEWS 1925 : Av. PASSERIF. (Acromyod.) Muscicapid.

Chloromunia MATHEWS 1923 : syn. de Lobospingus de VIS 1897.

Chloromyias OUSTALET $1880:$ Av. PASSERIF. (Acromyod.) Muscicapid.

Chloronerpes SWAINSON 1837: Av. PICIF. Picid. (Picin.).

Chloropeta SMITH 1847: syn. de Hippolais BALDERSTEIN 1827.

Chloropetella ROBERTS 1917: Av. PASSERIF. (Acromyod.) Sylviid.

Chlorophanes REICHENBACH 1853: Av. PASSERIF. (Acromyod.) Coerebid.

Chlorophis HALLOWELL 1857: syn. de Philothamnus SMITH 1847.

Chlorophoneus CABANIS 1851: Av. PASSERIF. (Acromyod.) Laniid.

Chlorophonia BONAPARTE 1851 : Av. PASSERIF. (Acromyod.) Tanagrid.

Chlorophthalmus BONAPARTE 1840: P.-CLUPEIF. (Myctophoid.) Chlorophthalmid.

Chloropipo CABANIS et HEINE 1859: Av. PASSERIF. (Mesomyod.) Piprid.

Chloropsis JARDINE et SELBY 1826: Av. PASSERIF. (Acromyod.) Pycnonotid.

Chloroptera : Av. PSITTACIF. Psittacid.

Chloroptyla SALVADORI 1871: syn. de Carduelis BRISSON 1760.

Chlorornis REICHENBACH 1850 : Av. PASSERIF. (Acromyod.) Timaliid.

Chloroscombrus GIRARD 1858: P.-PERCIF. (Percoid.) Carangid.

Chlorosoma WAGLER 1830:R.SQUAMAT. (Serpent.) Boigid.

Chlorospingus CABANIS 1851: Av. PASSERIF. (Acromyod.) Tanagrid.

Chlorostilbon GOULD 1853: Av. APODIF. Trochilid. 
Chlorotalpa ROBERTS 1924 : syn. d'Amblysomus POMEL 1848.

Chlorotesia DELACOUR 1942 : syn. d'Oligura HODGSON 1845.

Chlorothraupis RIDGWAY 1884: Av. PASSERIF. (Acromyod.) Tanagrid.

Chlorura SCLATER 1862: Av. PASSERIF. (Acromyod.) Ploceid. (Passerin.).

Chlorurisca SIMON 1921: syn. de Hypuroptila GOULD 1854.

Chlorurus SWAINSON 1839 : P.PERCIF. (Labroid.) Scarid.

Chodsigoa KASTASCHENKO 1907 : s./g. de Soriculus BLYTH 1854.

Choea : errat. pro Chloea JORDAN et SNYDER 1901.

Choerodon BLEEKER 1845: P.PERCIF. (Labroid.) Labrid.

Choeroichthys KAUP 1853: P.SYNGNATHIF. Syngnathid.

Choerojulis GILL 1862: P.-PERCIF. (Labroid.) Labrid.

Choeromys THOMAS 1922 : syn. de Thryonomys FITZINGER 1867.

Choeroniscus THOMAS 1928: M.CHIROPT. (Microchiropt.) Phyllostomatid. (Glossophagin.).

Choeronycteris LICHTENSTEIN 1844: M.-CHIROPT. (Microchiropt.) Phyllostomatid. (Glossophagin.).

Choerophryne van KAMPEN 1914 : syn. de Cophixalus BOETTEGER 1892.

Choeropithecus de BLAINVILLE 1839 : syn. de Papio MULLER 1773.

Choeroplotosus KNER 1866 : P.CYPRINIF. (Siluroid.) Plotosid.

Choerops RUPPELL 1852 : P.-PERCIF. (Labroid.) Labrid.

Choeropsis LEIDY 1853: M.-PARAX. (Suif.) Hippopotamid.

Choeropsodes : P.-PERCIF. (Labroid.) Labrid.

Choiropithecus REICHENBACH 1862 : syn. de Papio MULLER 1773 .

Choloepus ILLIGER 1811: M.EDENT. (Xenarthr.) Bradypodid.
Chologaster AGASSIZ 1853 : P.-AMBLYOPSIF. Amblyopsid.

Cholornis VERREAUX 1871: Av. PASSERIF. (Acromyod.) Paradoxiornithid.

Chondrestes : Av. PASSERIF. (Acromyod.) Fringillid.

Chondrochylus HECKEL 1843 : P.CYPRINIF. (Lyprinoid.) Cyprinid.

Chondrodactylus PETERS 1870 : R.-SQUAMAT. (Saur.) Geckonid.

Chondrohierax LESSON 1843: Av. FALCONIF. Falconid.

Chondroplites GILL 1862 : P.-PERCIF. (Stromateoid.) Stromateid.

Chondropython MEYER 1874: R.SQUAMAT. (Serpent.) Pythonid.

Chondrorhynchus HECKEL 1843 : P.-CYPRINIF. (Cyprinoid.) Cyprinid.

Chondrostoma AGASSIZ 1830 : P.CYPRINIF. (Cyprinoid.) Cyprinid.

Chonerhinus BLEEKER 1870 : P.TETRAODONTIF. (Tetraodontoid.) Tetraodontid.

Chonophorus POEY 1860: syn. d'Awaous STEINDACHNER 1860.

Chopinopsetta WHITLEY 1931 : P.PLEURONECTIF. (Pleuronectoid.) Bothid.

Chopraia PRASHAD et MUKERJI 1929: P.-CYPRINIF. (Cyprinoid.) Homalopterid.

Chorasia : R.-SQUAMAT. (Saur.) Agamid.

Chordeiles SWAINSON 1832: Av. CAPRIMULGIF. Caprimulgid. (Caprimulgin.).

Choregon MINDING 1832 : errat. pro Coregonus (LINNE 1758) de LACEPEDE 1803.

Choridactylodes GILCHRIST 1902 : P.-PERCIF. (Scorpaenoid.) Synancejid.

Choridactylus RICHARDSON 1848 : P.-PERCIF. (Scorpaenoid.) Synancejid.

Chorinectes : errat. pro Chironectes CUVIER 1817. 
Chorinemus CUVIER et VALENCIENNES 1831: P.-PERCIF. (Percoid.) Carangid.

Choriotis BONAPARTE : Av. RALLIF. Otidid.

Chorismodactylus GUNTHER 1860: P.-PERCIF. (Scorpaenoid.) Scorpaenid.

Chorismopelor CHEVEY 1927 : P.PERCIF. (Scorpaenoid.) Scorpaenid.

Chorismoschismus BRISOUT de BARNEVILLE 1846: P.-PERCIF. (Gobiesoxoid.) Gobiesocid.

Choristium GILL 1862 : P.-PERCIF. (Percoid.) Serranid.

Choristocalamus: R.-SQUAMAT. (Serpent.) Colubrid.

Choristodon SMITH 1849 : syn. de Choristocalamus.

Chorophilus BAIRD 1854: Amph. ANOUR. Hylid.

Chorotesia DELACOUR 1843: Av. PASSERIF. (Acromyod.) Timaliid. ou Sylviid.

Chorsomus : errat. pro Chrosomus RAFINESQUE 1820.

Chortomys THOMAS $1916: \mathrm{s} . / \mathrm{g}$. de Dendromus SMITH 1829.

Chorura REICHENBACH : errat. pro Chlorura SCLATER 1862.

Chotorhea BONAPARTE 1854: Av. PICIF. Capitonid.

Chriocephalus : Av. LARIF. Larid. (Larin.).

Chriolax JORDAN eî GILBERT 1879 : P.-PERCIF. (Scorpaenoid.) Triglid.

Chriolepis GILBERT 1891 : P.-PERCIF. (Gobioid.) Eleotrid.

Chriomitra LOCKINGTON 1879 : P. - PERCIF. (Scombroid.) Scombrid.

Chriomystax : P.-PERCIF. (Trachinoid.) Percophiid.

Chriope JORDAN 1878 : P.-CYPRINIF. (Cyprinoid.) Cyprinid.

Chriopeoides FOWLER 1939 : P.CYPRINIF. (Cyprinoid.) Cyprinid.

Chriopeons FOWLER 1916 : P.-CYPRINODONTIF. Cyprinodontid.

Christiella : P.-PERCIF. (Percoid.) Cichlid.
Christivomer HARKNESS 1942 : errat. pro Cristivomer GILL et JORDAN 1878.

Christomys SODY 1941: syn. de Rattus FISCHER 1803.

Chroeomys THOMAS 1916: s./g. d'Akodon MEYEN 1833.

Chromanthias WHITLEY 1935 : P.PERCIF. (Percoid.) Serranid.

Chromidotilapia $B O U L E N G E R$ 1898: P.-PERCIF. (Percoid.) Cichlid.

Chromileptes : vide Cromileptes SWAINSON 1839.

Chromis CU VIER 1814: P.-PERCIF. (Labroid.) Pomacentrid.

Chromogobius de BUEN 1930 : s./g. de Gobius LINNE 1758.

Chrosomus RAFINESQUE 1820 : P.-CYPRINIF. (Cyprinoid.) Catostomid.

Chrotogale THOMAS 1912 : M.-FISSIP. Viverrid. (Hemigalin.).

Chrotomys THOMAS 1895 : M.-RODENT. (Myomorph.) Murid. (Hydromyin.).

Chrotopterus PETERS 1865: M.CHIROPT. (Microchiropt.) Phyllostomatid. (Phyllostomatin.).

Chrysaeus SMITH 1839: syn. de Cuon HODGSON 1838.

Chrysailurus SEVERTZOV 1858: syn. de Felis LINNE 1758.

Chrysemys WAGLER nec GRAY 1844: R. - TESTUDIN. (Thecoph.) Emydid.

Chrysichthys BLEEKER 1858 : P.CYPRINIF. (Siluroid.) Bagrid.

Chrysiptera ZINCKEN 1817: P.PERCIF. (Labroid.) Pomacentrid.

Chrysipterus SWAINSON 1836 : syn. d'Iredaleichthys WHITLEY 1928.

Chrysobagrus BOULENGER 1899 : P.-CYPRINIF. (Siluroid.) Bagrid.

Chrysobatrachus : Amph. ANOUR. Rhacophorid.

Chrysoblephus SWAINSON 1839: P.-PERCIF. (Percoid.) Sparid.

Chrysochloris de LACEPEDE 1799: M.-INSECTIV. Chrysochlorid. (Chrysochlorin.). 
Chrysococcyx BOIE 1826 : Av. CUCULLIF. Cucullid.

Chrysocolaptes BLYTH 1843: Av. PICIF. Picid. (Picin.).

Chrysocyon SMITH 1839 : M.-FISSIP. Canid. (Canin.).

Chrysoenas BONAPARTE 1854: Av. COLUMBIF. Columbid. (Treronin.).

Chrysolampis BOIE 1831: Av. APODIF. Trochilid.

Chrysolophus GRAY 1834: Av. PHASIANIF. Phasianid. (Phasianin.).

Chrysomitridops WILLSON 1889 : Av. PASSERIF. (Acromyod.) Fringillid. (Fringillin.).

Chrysomitris BOIE 1828 : Av. PASSERIF. (Acromyod.) Fringillid. (Fringillin.).

Chrysomma HODGSON 1845: Av. PASSERIF. (Acromyod.) Timaiud.

Chrysonycteris GRAY 1866 : syn. d'Hipposideros GRAY 1831.

Chrysopelea BOIE 1826 : R.-SQUAMAT. (Serpent.) Boigid.

Chrysoperca FOWLER 1907 : P.PERCIF. (Percoid.) Serranid.

Chrysophekadion BLEEKER 1860 : P.-CYPRINIF. (Cyprinoid.) Cyprinid.

Chrysophlegma GOULD 1850 : Av. PICIF. Picid. (Picin.).

Chrysophrys CUVIER et VALENCIENNES 1830: P.-PERCIF. (Percoid.) Sparid.

Chrysopicus MALHERBE 1849 : Av. PICIF. Picid. (Picin.).

Chrysopteron JENTINK $1910: s . / g$. de Myotis KAUP 1829.

Chrysoptilopicus $M A L H E R B E$ 1845: Av. PICIF. Picid. (Picin.).

Chrysoptilus SWAINSON 1832 : Av. PICIF. Picid. (Picin.).

Chrysospalax GILL 1883: M.-INSECTIV. Chrysochlorid. (Chrysochlorin.).

Chrysotrichia BROOM : syn. d'Amblysomus POMEL 1848.

Chrysothrix KAUP 1835: syn. de Saimiri VOIGT 1831.

Chrysotis SWAINSON 1837: Av. PSITTACIF. Psittacid.
Chrysotosus de LACEPEDE 1802 : P.-LAMPRIDIF. Lampridid.

Chrysotreron BONAPARTE 1854: Av. COL U M BI F. Columbid. (Treronin.).

Chrysotrogon RIDGWAY 1911: Av. TROGONIF. Trogonid.

Chrysuronia BONAPARTE 1850 : Av. APODIF. Trochilid.

Chthamalopteryx OGILBY 1888 : P.-PERCIF. (Percoid.) Leiognathid.

Chthonerpeton PETERS 1879 : Amph. APOD. Caeciliid.

Chuanchia HERTZENSTEIN 1891 : P.-CYPRINIF. (Cyprinoid.) Cyprinid.

Chubbia MATHEWS 1913: Av. CHARADRIIF. Charadriid. (Scolopacin.).

Chunga HARTLAUB 1860 : errat. pro Chunya SHARPE.

Chunya SHARPE : Av. RALLIF. Cariamid.

Ciccaba WAGLER 1832: Av. STRIGIF. Strigid. (Bubonin.).

Cichla SCHNEIDER 1801 : P.-PERCIF. (Percoid.) Cichlid.

Cichladusa PETERS 1863: Av. PASSERIF. (Acromyod.) Turdid. (Phoenicurin.).

Cichlasoma SWAINSON 1839 : P.PERCIF. (Percoid.) Cichlid.

Cichlaurus SWAINSON 1839 : syn. de Cichlasoma S. 1839.

Cichlherminia BONAPARTE 1854: Av. PASSERIF. (Acromyod.) Mimid.

Cichlomyia OBERHOLSER 1905: Av. PASSERIF. (Acromyod.) Muscicapid.

Cichlopasser BONAPARTE 1855: Av. PASSERIF. (Acromyod.) Frinaillid.

Cichlops MULLER et TROSCHEL 1849 : P.-PERCIF. (Percoid.) Serranid.

Cichlopsis CABANIS 1851: Av. PASSERIF. (Acromyod.) Turdid. (Myadestin.).

Cichlornis MAYR 1933: Av. PASSERIF. (Acromyod.) Timaliid.

Cichloselys BONAPARTE 1854: Av. PASSERIF. (Acromyod.) Turdid. 
Ciciqna GRAY 1825 : R.-SQUAMAT. (Saur.) Gerrhosaurid.

Cicinnurus VIEILLOT 1816: Av. PASSERIF. (Acromyod.) Paradiseid. (Paradisein.).

Cicla WALBAUM 1792 : P.-PERCIF. (Labroid.) Labrid.

Ciclorhis : errat. pro Cyclarhis SWAINSON 1824.

Ciconia LINNE 1758: Av. ARDEIF. Ciconid.

Ciliata COUCH 1832: P.-GADIF. Gadid.

Cile : P.-CLUPEIF. (Clupeoid.) Clupeid.

Cilus DELFIN 1900: P.-PERCIF. (Percoid.) Sciaenid.

Cinathisma HULL 1916: Av. PROCELLARIF. Procellariid.

Cinclocerthia GRAY 1840: Av. PASSERIF. (Acromyod.) Mimid.

Cinclodes GRAY 1840 : Av. PASSERIF. (Mesomyod.) Furnariid.

Cinclorhamphus GOULD 1838 : Av. PASSERIF. (Acromyod.) Paradoxiornithid.

Cinclosoma VIGORS et HORSFIELD 1826: Av. PASSERIF. (Mesomyod.) Dendrocolaptid.

Cinclus BORKHAUSEN 1797: Av. PASSERIF. (Acromyod.) Cinclid.

Cinetodus OGILBY 1898 : P.-CYPRINIF. (Siluroid.) Bágrid.

Cingla FLEMMING 1822 : P.-PERCIF. (Percoid.) Percid.

Cingulogobius HERRE 1927: syn. de Pleurogobius.

Cinixys BELL 1827 : R.-TESTUDIN. (Thecoph.) Testudinid.

Cinnamopterus BONAPARTE 1823: Av. PASSERIF. (Acromyod.) Sturnid. (Graculin.).

Cinnamopteryx REICHENOW 1886: Av. PASSERIF. (ACromyod.) Ploceid.

Cinnycerthia LESSON 1844: Av. PASSERIF. (Acromyod.) Troglodytid.

Cinnyricinclus LESSON 1840: Av. PASSERIF. (Acromyod.) Sturnid. (Graculin.).

Cinnyris CUVIER 1817: Av. PAS.
SERIF. (Acromyod.) Nectariniid.

Cinosternum WAGLER 1830 : syn. de Kinosternon SPIX 1824.

Circaëtus VIEILLOT 1816: Av. FALCONIF. Falconid. (Aquilin.).

Circosaura : errat. pro Cricosaura GUNDLACH et PETERS 1864.

Circus de LACEPEDE 1799: Av. FALCONIF. Falconid. (Circin.).

Ciridops NEWTON 1892: Av. PASSERIF. (Acromyod.) Drepanidid.

Cironomys SODY 1941: syn. de Rattus FISCHER 1803.

Cirrinidesmus BONAPARTE 1856 : syn. de Charadrius LINNE 1758.

Cirrhichthys : errat. pro Cirrhitichthys BLEEKER 1870.

Cirrhigaleus TANAKA 1912: P.SQUALIF. (Squalioid.) Scymnorhinid.

Cirrhilabrus TEMMINCK et SCHLEGEL 1845: P.-PERCIF. (Labroid.) Labrid.

Cirrhimens (GILL 1862) BLEEKER 1876 : P.-PERCIF. (Percoid.) Sciaenid.

Cirrhimuraena KAUP 1856: P.A NGUILLIF. (Congroid.) Ophichthyid.

Cirrhina CUVIER et VALENCIENNES 1842 : P.-CYPRINIF. (Cyprinoid.) Cyprinid.

Cirrhinichthys BLEEKER 1859 : P.-CYPRINIF. (Cyprinoid.) Cyprinid.

Cirrhinus OKEN 1817 : P.-CYPRINIF. (Cyprinoid.) Cyprinid.

Cirrhipipra BONAPARTE 1850 : syn. de Teleonema REICHEN$B A C H 1850$.

Cirrhipectes : vide Cirripectes SWAINSON 1839.

Cirrhites FRORIEP 1806 : syn. de Cirrhitus de LACEPEDE 1803.

Cirrhitichthys BLEEKER 1870 : P. PERCIF. (Percoid.) Cirrhitid.

Cirrhitoidea JENKINS 1904: P.PERCIF. (Percoid.) Cirrhitid.

Cirrhitopsis GILL 1862 : P.-PERCIF. (Percoid.) Cirrhitid. 
Cirrhitus de LACEPEDE 1803 : P.PERCIF. (Percoid.) Cirrhitid.

Cirrhoscyllium SMITH 1913: P.GALEIF. (Isuroid.) Orectolobid.

Cirricaecula SCHULTZ 1953 : P.ANGUILLIF. (Congroid.) Ophichthyid.

Cirrinectus SWAINSON 1839 : P.PERCIF. (Blennioid.) Blenniid.

Cirriscyllium OGILBY 1908: syn. de Cirrhoscyllium SMITH 1913.

Cirropicus STRESEMANN 1912 : Av. PICIF. Picid. (Picin.).

Cisco JORDAN et EVERMANN 1911: P.-CLUPEIF. (Salmonoid.) Salmonid.

Cissa BOIE 1826: Av. PASSERIF. (Acromyod.) Corvid.

Cissolopha BONAPARTE 1850: Av. PASSERIF. (Acromyod.) Corvid.

Cissopica DELACOUR 1927: Av. PASSERIF. (Acromyod.) Tanagrid.

Cisticola KAUP 1829 : Av. PASSERIF. (Acromyod.) Sylviid. ou Timaliid.

Cistothorus CABANIS 1851: Av. PASSERIF. (Acromyod.) Troglodytid.

Cistudo DUMERIL et BIBRON : syn. de Terrapene MERREM 1820.

Cistudo SCHNEIDER : syn. d'Emys DUMERIL 1806.

Cistugo THOMAS 1912: M.-CHIROPT. (Microchiropt.) Vespertilionid. (Vespertilionin.).

Citellus OKEN 1816 : M.-RODENT. (Sciuromorph.) Sciurid. (Sciurin.).

Citharichthys BLEEKER 1862 : P.PLEURONECTIF. (Pleuronectoid.) Bothid.

Citharidium BOULENGER 1902: P.-C Y P R I N I F. (Characoid.) Characid.

Citharinus CUVIER 1817 : $P .-C Y$ PRINIF. (Characoid.) Characid.

Citharoedus KAUP 1860 : P.-PERCIF. (Percoid.) Chaetodontid.

Citharoides HUBBS 1915: P.-PLEU-
RONECTIF. (Pleuronectoid.) Bothid.

Citharus ROSE 1793 : P.-PLEURONECTIF. (Pleuronectoid.) Bothid.

Cittocinchla SCLATER et SAUNDERS 1884: Av. PASSERIF. (Acromyod.) Turdid. (Phoenicurin.).

Cittura KAUP 1848 : Av. CORACIADIF. Alcedinid.

Citula CUVIER 1817 : P.-PERCIF. (Percoid.) Carangid.

Civettictis POCOCK 1915: M.-FISSIP. Viverrid. (Viverrin.).

Cladobates CUVIER 1824 : syn. de Tupaia RAFFLES 1821.

Cladorhynchus GRAY 1840: Av. CHARADRIIF. Charadriid. (Recurvirostrin.).

Cladoselache DEAN 1894 : syn. de cutamydoselachus $G A R M A N$ 1884.

Clais SCLATER 1862 : Av. APODIF. Trochilid.

Clamator BLYTH 1849 : Av. CUCULLIF. Cucullid.

Clamosocircus SWANN 1925:Av. FALCONIF, Falconid. (Circin.).

Clangula OKEN 1817: Av. ANATIF. Anatid. (Anatin.).

Clara GILL 1862 : P.-PERCIF. (Percoid.) Leiognathid.

Clarasilurus : errat. pro Clarisilurus FOWLER 1938.

Claravis OBERHOLSER 1899:Av. COLUMBIF. Columbid. (Columbin.).

Clariallabes BOULENGER 1900: P.-CYPRINIF. (Siluroid.) Clariid.

Clarias GRONOV 1781 (nec SCOPOLI 1777) : P.-CYPRINIF. (Siluroid.) Clariid.

Claricola JORDAN et EVERMANN 1896 : P.-PERCIF. (Percoid.) Etheostomid.

Clariger JORDAN et SNYDER 1901 : P.-PERCIF. (Gobioid.) Gobiid.

Clarisilurus FOWLER 1938 : P.-CYPRINIF. (Siluroid.) Silurid.

Clarkina JORDAN et EVERMANN 
1927 : P.-CYPRINIF. (Cyprinoid.) Cyprinid.

Clarotes KNER 1855 : P.-CYPRINIF. (Siluroid.) Bagrid.

Claudia HARTERT 1892 : Av. APODIF. Apodid.

Claudius COPE 1865 : R.-TESTUDIN. (Thecoph.) Kinosternid.

Claviglis JENTINK 1888 : s./g. de Graphiurus SMUTS 1832.

Cleidoporus : errat. pro Cleidopus de VIS 1832.

Cleidopus de VIS 1832 : P.-BERYCIF. Monocentrid.

Cleisthenes JORDAN et STARKS 1904: P.-PLEURONECTIF. (Pleuronectoid.) Pleuronectid.

Clelia FITZINGER 1826 : syn. de Peudoboa SCHNEIDER 1801.

Clemmys WAGLER 1830 : R.-TESTUDIN. (Thecoph.) Emydid.

Clepticus CUVIER 1829: P.-PERCIF. (Labroid.) Labrid.

Clethrionomys TILESIUS 1850 : M.-RODENT. (Myomorph.) Microtid.

Clevelandia EIGENMANN 1888: P.-PERCIF. (Gobioid.) Gobiid.

Clibanornis SCLATER et SALVIN 1873: Av. PASSERIF. (Mesomyod.) Furnariid.

Clibanus GISTEL 1848 : Av. PASSERIF. (Mesomyod.) Furnariid.

Clidoderma BLEEKER 1862: $P$.PLEURONECTIF. (Pleuronectoid.) Pleuronectid.

Climacocercus CABANIS 1844: syn. de Clamosocircus $S W A N N$ 1925 .

Climacocercus BERTONI 1901: Av. PASSERIF. (Mesomyod.) Cotingid.

Climacoporus BARNARD 1935 : P.PERCIF. (Cottoid.) Cottid.

Climacteris TEMMINCK 1820: Av. PASSERIF. (Acromyod.) Certhiid. ou Sittid.

Clinocottus GILL 1861 : P.-PERCIF. (Cottoid.) Cottid.

Clinodon REGAN 1920: P.-PERCIF. (Percoid.) Cichlid.

Clinoides GILCHRIST et THOMPSON 1908: P.-PERCIF. (Blennioid.) Clinid.
Clinoporus BARNARD 1927: P.PERCIF. (Blennioid.) Clinid.

Clinostomus GIRARD 1856 : P.-CYPRINIF. (Cyprinoid.) Cyprinid.

Clinus CUVIER 1817 : P.-PERCIF. (Blennioid.) Clinid.

Cliola GIRARD 1856 : P.-CYPRINIF. (Cyprinoid.) Cyprinid.

Clitoceyx : errat. pro Clytoceyx SHARPE 1880.

Clivicola FORSTER 1817 : syn. de Riparia FORSTER 1817.

Clodalus RAFINESQUE 1820 : P.CLUPEIF. (Notopteroid.) Hiodontid.

Clodipterus RAFINESQUE 1815 : P.-PERCIF. (Percoid.) Serranid.

Cloeotis THOMAS 1901: M.-CHIROPT. (Microchiropt.) Hipposiderid.

Cloroscombrus : errat. pro Chloroscombrus GIRARD 1858.

Clotho GRAY 1840 : syn. de Bitis GRAY 1842.

Clunalosa BLEEKER 1870 : P.CLUPEIF. (Clupeoid.) Clupeid.

Clupanodon de LACEPEDE 1803 : P.-CLUPEIF. (Clupeoid.) Dorosomid.

Clupea LINNE 1758 : P.-CLUPEIF. (Clupeoid.) Clupeid.

Clupeichthys BLEEKER 1855 : P.CLUPEIF. (Clupeoid.) Clupeid.

Cluveocharax PELLEGRIN 1926: syn. de Clupeopetersius PELLEGRIN 1928.

Clupeocharax PEARSON 1924 : P.CYPRINIF. (Characoid.) Characid.

Cluneoides BLEEKER 1851: P.CLUPEIF. (Clupeoid.) Clupeid.

Clupeonella KESSLER 1877 : P.CLUPEIF. (Clupeoid.) Clupeid.

Clupeonia CUVIER et VALENCIENNES 1847: P.-CLUPEIF. (Clupeoid.) Clupeid.

Clupeopetersius PELLEGRIN 1928: P.-C Y PRINIF. (Characoid.) Characid.

Cluvisoma SWAINSON 1838: P. CYPRINIF. (Siluroid.) Silurid.

Clupisudis SWAINSON 1839: P.- 
CLUPEIF. (Osteoglossoid.) Osteoglossid.

Clymene GRAY 1864: syn. de Stenella GRAY 1866.

Clymenia GRAY 1868 : syn. de Stenella GRAY 1866.

Clyomys THOMAS 1916: M.-RODENT. (Caviomorph.) Echimyid. (Echimyin.).

Clypeobarbus FOWLER 1936 : s./g. de Barbus CUVIER 1817.

Clypeocottus AYRES 1854 : P.-PERCIF. (Cottoid.) Cottid.

Clypicterus BONAPARTE 1850: Av. PASSERIF. (Acromyod.) Icterid. (Cacicin.).

Clytoceyx SHARPE 1880 : Av. CORACIADIF. Alcedinid.

Clytoctantes ELLIOT 1870: Av. PASSERIF. (Mesomyod.) Formicariid. (Thamnophilin.).

Clytolaema GOULD 1853: Av. APODIF. Trochilid.

Clytomyias SHARPE 1879: Av. PASSERIF. (Acromyod.) Muscicapid.

Clytorhynchus ELLIOT 1870: Av. PASSERIF. (Acromyod.) Muscicapid.

Clytospiza SHELLEY 1896: Av. PASSERIF. (Acromyod.) Ploceid. (Estrildin.).

Cnemarchus RIDGWAY 1907: Av. PASSERIF. (Mesomyod.) Tyrannid.

Cnemaspis STRAUCH 1887: R.SQUAMAT. (Saur.) Geckonid.

Cnemidophorus WAGLER 1830 : R.-SQUAMAT. (Saur.) Tejid.

Cnemiornis OWEN 1865 : Av. ANATIF. Anatid.

Cnemonax BRODKORB 1936: Av. PASSERIF. (Mesomyod.) Tyrannid.

Cnemophilus de VIS 1890: Av. PASSERIF. (Acromyod.) Paradiseid. (Paradisein.).

Cnemophora : R.-SQUAMAT. (Serpent.) Colubrid.

Cnemotriccus HELLMAYR 1927: Av. PASSERIF. (Mesomyod.) Tyrannid.

Cnephaeus KAUP 1829 : syn. d'Eptesicus RAFINESQUE 1820.
Cnestrodon GARMAN 1895 : P.-CYPRINODONTIF. Poeciliid.

Cnestrostoma REGAN 1920: P.PERCIF. (Percoid.) Cichlid.

Cnidoglanis GUNTHER 1864: P.CYPRINIF. (Siluroid.) Plotosid.

Cnidon MULLER et TROSCHEL 1849 : P.-PERCIF. (Percoid.) Serranid.

Cnipodectes SCLATER et SALVIN 1873: Av. PASSERIF. (Mesomyod.) Tyrannid. (Tyrannin.).

Cnipolegus STRICKLAND 1841: syn. de Knipolegus BOIE 1824.

Cobitichthys BLEEKER 1859 : P.CYPRINIF. (Cyprinoid.) Cobitid.

Cobitoglanis FOWLER 1914 : P.CYPRINIF. (Siluroid.) Trichomycterid.

Cobitinula HANKO 1925: P.-CYPRINIF. (Cyprinoid.) Cobitid.

Cobitis ARTEDI 1738 : P.-CYPRINIF. (Cyprinoid.) Cobitid.

Cobitophis MYERS 1927: P.-CYPRINIF. (Cyprinoid.) Cobitid.

Cobitopsis POMEL 1853 : P.-CYPRINIF. (Cyprinoid.) Cobitid.

Cobra LAURENTI 1768: syn. de Naja LAURENTI 1768.

Coccia GUNTHER 1864 : syn. d'Ichthyococcus BONAPARTE 1841.

Coccogenia COCKERELL et CALLAWAY 1909: P.-CYPRINIF. (Cyprinoid.) Cyprinid.

Coccolarynx REICHENBACH 1851: Av. CORACIADIF. Meropid.

Coccolus COCCO 1844 : P.-GADIF. Macrurid.

Coccopygia REICHENBACH 1862 : Av. PASSERIF. (Acromyod.) Ploceid.

Coccorella ROULE 1929: syn. d'Evermanella FOWLER 1901.

Coccothraustes BRISSON 1760 : Av. PASSERIF. (Acromyod.) Fringillid. (Coccothrautin.).

Coccotis JORDAN 1882 : P.-CYPRINIF. (Cyprinoid.) Cyprinid.

Coccotropsis BARNARD 1927 : P.PERCIF. (Scorpaenoid.) Scorpaenid.

Coccotropus (KAUP 1858) BLEEKER 1876 : P.-PERCIF. (Scorpaenoid.) Aploactid. 
Coccystes GLOGER 1833 : Av. CUCULLIF. Cucullid.

Coccyzus VIEILLOT 1816 : Av. CUCULLIF. Cucullid.

Cochlearius BRISSON 1760: Av. ARDEIF. Ardeid.

Cochleavius : errat. pro Cochlearius BRISSON 1760.

Cochlefelis WHITLEY 1941: P.CYPRINIF. (Siluroid.) Bagrid.

Cochlioceps WHITLEY 1943 : P.PERCIF. (Gobiesoxoid.) Gobiesocid.

Cochliodon (HECKEL) GUNTHER 1864 : P.-CYPRINIF. (Siluroid.) Loricariid.

Cochliophagus DUMERIL et BIBRON 1853: R.-SQUAMAT. (Serpent.) Colubrid.

Cochlognathus BAIRD et GIRARD 1854: P.-CYPRINIF. (Cyprinoid.) Cyprinid.

Cochoa HODGSON 1836 : Av. PASSERIF. (Acromyod.) Prionopid.

Cochranella : Amph. ANOUR. Leptodactylid.

Cociella WHITLEY 1940 : P.-PERCIF. (Scorpaenoid.) Platycephalid.

Cocius JORDAN et HUBBS 1925: syn. de Cociella W. 1940.

Cocliophagus : errat. pro Cochliophagus DUMERIL et BIBRON 1853.

Cocotropus KAUP 1858 : vide Coccotropus (K. 1858) BLEEKER 1876.

Codoma GIRARD 1856: syn. de Notropis RAFINESQUE 1818.

Coecilia : errat. pro Caecilia LINNE 1758.

Coeciliophis KAUP 1856 : P.-ANGUILLIF. (Congroid.) Ophichthyid.

Coecula CUVIER 1817 : errat. pro Caecula VAHL 1794.

Coeligena LESSON 1833 : Av. APODIF. Trochilid.

Coelocephalus GILBERT et CRAMMER 1897: P.-GADIF. Macrurid.

Coelodactylus : errat. pro Coleodactylus PARKER 1926.

Coelodus : Av. PASSERIF. (Acromyod.) Pycnonotid.
Coelogenus CUVIER 1807 : syn. de Cuniculus BRISSON 1762.

Coelomys THOMAS 1915: M.-RODENT. (Myomorph.) Murid. (Murin.).

Coelonotus PETERS 1855 : P.-SYNGNATHIF. Syngnathid.

Coelopeltis WAGLER 1830 : syn. de Malpolon FITZINGER 1826.

Coelophyllus PETERS 1871: syn. de Rhinolophus de LACEPEDE 1799.

Coelops BLYTH 1848: M. - CHIROPT. (Microchiropt.) Hipposiderid.

Coelorhynchus GIORNA 1809 : P.GADIF. Macrurid.

Coelotis : M.-CHIROPT. (Microchiropt.) Hipposiderid.

Coendou de LACEPEDE 1799 : M.RODENT. (Caviomorph.) Erethizonthid. (Erethizonthin.).

Coendu LESSON 1827: syn. de Coendou de LACEPEDE 1799.

Coenocorywha GRAY 1855: Av. CHARADRIIF. Charadriid. (Scolopacin.).

Coenotriccus SCLATER 1888: Av. PASSERIF. (Mesomyod.) Tyrannid.

Coephloeus CABANIS et HEINE : Av. PICIF. Picid. (Picin.).

Coereba VIEILLOT 1807 : Av. PASSERIF. (Acromyod.) Coerebid.

Coetomys GRAY 1864: syn. de Cryptomys GRAY 1864.

Cogia WALLAVE 1876 : emend. pro Kogia GRAY 1846.

Cogrus RAFINESQUE 1810 : P.-ANGUILLIF. (Congroid.) Ophichthyid.

Coilia GRAY 1831: P.-CLUPEIF. (Clupeoid.) Engraulid.

Coius HAMILTON-BUCHANAN 1822: P.-PERCIF. (Percoid.) Serranid.

Cokeridia MEEK et HILDEBRAND 1928: P. - PERCIF. (Trachinoid.) Dactyloscopid.

Colaptes SWAINSON 1825 : Av. PICIF. Picıd. (Picin.).

Colefaxia WHITLEY $1935: s . / g$ de Neoplatycephalus CASTELNAU 1872. 
Coleia MATHEWS 1931: Av. PASSERIF. (Acromyod.) Meliphagid.

Colena MATHEWS 1931: syn. de Coleia MATHEWS 1931.

Coleodactylus PARKER 1926 : R.SQUAMAT. (Saur.) Geckonid.

Coleonyx GRAY 1845: R.-SQUAMAT. (Saur.) Geckonid.

Coleosmaris NORMAN 1931: P.PERCIF. (Percoid.) Maenid.

Coleotropis MYERS 1942 : P.-MUGILIF. Atherinid.

Coleura PETERS 1867: M.-CHIROPT. (Microchiropt.) Emballonurid.

Colibri SPIX 1824: Av. APODIF. Trochilıd.

Colinus GOLDFUSS 1820: Av. PHASIANIF. Phasianid. (Phasianin.).

Coliostruthus SUNDEVALL 1849 : Av. PASSERIF. (Acromyod.) Fringillid.

Colisa CUVIER et VALENCIENNES 1831 : P. - PERCIF. (Anabantoid.) Anabantid.

Coliscus COPE 1872 : P.-CYPRINIF. (Cyprinoid.) Cyprinid.

Colistium NORMAN 1926: P.PLEURONECTIF. (Pleuronectoid.) Pleuronectid.

Colius BRISSON 1760 (nec MOEH$R I N G 1758)=A v . C O L I I F . C o-$ liid.

Coliuspasser RUPPELL 1840: Av. PASSERIF. (Acromyod.) Ploceid. (Viduin.) (serait syn. de Penthethria CABANIS 1847).

Collettia GOODE et BEAN 1896 : P.-C LU P E I F. (Myctophoid.) Myctophiid.

Colletoptera ROBERTS 1922: Av. APODIF. Apodid.

Collichthys GUNTHER 1860: P.PERCIF. (Percoid.) Sciaenid.

Collocalia GRAY 1840: Av. APODIF. Apodid.

Collorhabdium SMEDLEY 1932: R.-SQUAMAT. (Serpent.) Colnbrid.

Colluricinchla VIGORS et HORSFIELD 1826: Av. PASSERIF. (Acromyod.) Prionopid.
Collybus SNYDER 1904 : P.-CORYPHAENIF. Bramid.

Colobates REY 1872 : errat. pro Calobates KAUP 1829.

Colobodactylus AFRANIO do AMA$R A L$ 1932: R.-SQUAMAT. (Saur.) Tejid.

Coloborhynchus OWEN 1874 : R.SQUAMAT. (Saur.) Tejid.

Colobosaura BOULENGER 1887 : R.-SQUAMAT. (Saur.) Tejid.

Colobotis BRANDT 1844: syn. de Citellus OKEN 1816.

Colobus ILLIGER 1811: M.-PRIMAT. (Simioid.) Cercopithecid. (Colobin.).

Coloconger ALCOCK 1889 : P.-AN. GUILLIF. (Congroid.) Congrid.

Colocopus GILL 1884 : syn. de Paracanthurus BLEEKER 1863.

Coloeus KAUP 1829: Av. PASSERIF. (Acromyod.) Corvid.

Cololabis GILL 1896 : P.-BELONIF. (Belonoid.) Scombresocid.

Colomesus GILL 1885: P.-TETRAODONTIF. (Tetraodontoid.) Tetraodontid.

Colomys THOMAS et WROUGHTON 1907: M.-RODENT. (Myomorph.) Murid. (Murin.).

Colopteryx RIDGWAY 1888: Av. PASSERIF. (Mesomyod.) Tyrannid. (Spizitornithin.).

Coloptychon THIEN 1949 : R.SQUAMAT. (Saur.) Anguid.

Colopus PETERS 1869 : R.-SQUAMAT. (Saur.) Geckonid.

Colossoma EIGENMANN 1903 : P.CYPRINIF. (Characoid.) Characid.

Colosthetus COPE 1866: Amph. ANOUR. Ranid.

Colpichthys HUBBS 1918 : P.-MUGILIF. Atherinid.

Colpochelys GARMAN 1880: R.TESTUDIN. (Thecoph.) Cheloniid.

Colpoglossus BOULENGER 1904: Amph. ANOUR. Microhylid.

Colpognathus KLUNZINGER 1879: syn. d'Othos CASTELNAU 1875 . 
Colpophis SMITH 1926: R.-SQUAMAT. (Serpent.) Hydrophiid.

Coluber LINNE 1758: R.-SQUAMAT. (Serpent.) Colubrid.

Columba LINNE 1758: Av. COLUMBIF. Columbid. (Columbin.).

Columbia EIGENMANN 1892 : P.PERCOPSIF. Percopsid.

Columbigallina OKEN 1817: Av. COLUMBIF. Columbid. (Columbin.).

Columbina v. SPIX 1825 : syn. de Columbula BONAPARTE 1854.

Columbula BONAPARTE 1854:Av. COLUMBIF. Columbid. (Columbin.).

Colus WAGNER 1844 : syn. de Saiga GRAY 1843.

Colymbus BRISSON : syn. de Podiceps LATHAM 1787.

Colymbus KLUK 1779 : syn. de Podiceps LATHAM 1787.

Colymbus LINNE 1758: Av. COLYMBIF. Colymbid.

Comaroptera : errat. pro Camaroptera SUNDEVALL 1850.

Comastes FITZINGER 1870: syn. de Myotis KAUP 1829.

Comatibis REICHENBACH 1852 : Av. ARDEIF. Plataleid. (Threskiornithin.).

Comephorus de LACEPEDE 1800 : P.-PERCIF. (Cottoid.) Cottid.

Cometornis BANGS et PENARD 1921: Av. PASSERIF. (Mesomyod.) Tyrannid.

Commandorella TARA NETS et ANDRYASHEV 1935 : P.-PERCIF. (Blennioid.) Zoarcid.

Comopithecus ALLEN 1925: s./g. de Papio MULLER 1773.

Compsocoma CABANIS 1851: Av. PASSERIF. (Acromyod.) Tanagrid.

Compsomidiama CHABANAUD 1951: P.-PLEURONECTIF. (Soleoid.) Cynoglossid.

Compsophis MOCQUARD 1894 : R.SQUAMAT. (Serpent.) Colubrid.

Compsothlypis CABANIS 1851: Av. PASSERIF. (Acromyod.) Parulid.

Compsothraupis RICHEMOND
1915: Av. PASSERIF. (Acromyod.) Tanagrid.

Compsotis HEINE 1890 : Av. RALLIF. Otidid.

Compsura EIGENMANN 1915 : P.CYPRINIF. (Characoid.) Characid.

Comura PETERS : s./g. de Saccopteryx ILLIGER 1811.

Conchognathus COLLETT 1889 : syn. de Simenchelys (GILL) GOODE et BEAN 1879.

Condylura ILLIGER 1811: M.-INSECTIV. Talpid. (Condylurin.).

Conepatus GRAY 1837 : M.-FISSIP. Mustelid. (Mephitin.).

Conessius : P.-CYPRINIF. (Cyprinoid.) Cyprinid.

Conger (KLEIN) SCHAEFFER 1760 : P.-ANGUILLIF. (Congroid.) Congrid.

Congermuraena KAUP 1856 : P.ANGUILLIF. (Congroid.) Congrid.

Congerodon KAUP 1856 : P.-ANGUILLIF. (Congroid.) Congrid.

Congiopodus PENY 1811: syn. de Congiopus REGAN 1913.

Congiopus REGAN 1913: P.-PERCIF. (Scorpaenoid.) Congiopid.

Congramonus FOWLER 1906 : P.-

Congrellus OGILBY 1898: P.-ANGUILLIF. (Congroid.) Congrid.

Congresox GILL 1890 : P.-ANGUILLIF. (Congroid.) Congrid.

Congrhynchus FOWLER 1934 : P.ANGUILLIF. (Congroid.) Congrid.

Congrina JORDAN et HUBBS 1925: P.-ANGUILLIF. (Congroid.) Congrid.

Congriscus JORDAN et HUBBS 1925: P. - ANGUILLIF. (Congroid.) Congrid.

Congrogadoides BORODIN 1933 : P.-PERCIF. (Blennioid.) Congrogadid.

Congrogadus GUNTHER 1862 : P.PERCIF. (Blennioid.) Congrogadid.

Congromuraena GUNTHER 1870 : syn. de Congermuraena KAUP 1856.

Congrosoma GARMAN 1899 : P.- 
ANGUILLIF. (Congroid.) Congrid.

Congrus RICHARDSON 1845 : P.ANGUILLIF. (Congroid.) Congrid.

Conidens BRIGGS 1955: P.-PERCIF. (Gobiesoxoid.) Gobiesocid.

Conilurus OGILBY 1838: M.-RODENT. (Myomorph.) Murid. (Murin.).

Coniophanes (HALLOWELL) COPE $1860:$ R.-SQUAMAT. (Serpent.) Colubrid.

Conirostrum d'ORBIGNY et LAFRESNAYE 1838: Av. PASSERIF. (Acromyod.) Coerebid.

Connochaetes LICHTENSTEIN 1814 : M.-PARAX. (Solenodont.) Bovid. (Alcelaphin.).

Conocara JORDAN et EVERMANN 1896 : P.-CLUPEIF. (Alepocephaloid.) Alepocephalid.

Conodon CUVIER et VALENCIENNES 1830 : P.-PERCIF. (Percoid.) Lutjanid.

Conolophus FITZINGER 1843 : R.SQUAMAT. (Saur.) Iguanid.

Conophis PETERS 1860 : R.-SQUAMAT. (Serpent.) Boigid.

Conopoderas BILLBERG 1828: Av. PASSERIF. (Acromyod.) Sylviid.

Conopophaga VIEILLOT 1816: Av. PASSERIF. (Mesomyod.) Conopophagid.

Conopoderma F $O$ WLER 1934 : s./g. de Poroderma SMITH 1838.

Conopsis GUNTHER 1858 : R.SQUAMAT. (Serpent.) Colubrid.

Conorhynchos BLEEKER 1863 : P.CYPRINIF. (Siluroid.) Bagrid.

Conorhynchus GILL 1861 : P.-CLUPEIF. (Elopoid.) Albulid.

Conostoma HODGSON 1842: Av. PASSERIF. (Acromyod.) Paradoxiornithid.

Conothoa LYON 1904: syn. d'Ochotona LINK 1795.

Conothraupis SCLATER 1880: Av. PASSERIF. (Acromyod.) Tanagrid.

Constrictor LAURENTI 1768 : syn. de Boa LINNE 1758.
Conta HORA 1950 : P.-CYPRINIF, (Siluroid.) Bagrid.

Contia BAIRD et GIRARD 1853: R.-SQUAMAT. (Serpent.) Colubrid. (serait syn. de Cyclophis GUNTHER 1858).

Contopus CABANIS 1855 : Av. PASSERIF. (Mesomyod.) Tyrannid. (Tyrannin.).

Contusus WHITLEY 1947 : P.-TETRAODONTIF. (Tetraodontoid.) Tetraodontid.

Conuropsis SALVADORI 1891: Av. PSITTACIF. Psittacid.

Conurus KUHL 1820: Av. PSITTACIF. Psittacid.

Cookeolus FOWLER $1928: s . / g$. de Priacanthus OKEN 1817.

Cookilaria BONAPARTE 1856 : Av. PROCELLARIF. Procellariid.

Copea STEINDACHNER 1864: Amph. ANOUR. Microhyud.

Copeia : P.-CLUPEIF. (Clupeoid.) Engraulid.

Copeina FOWLER 1906 : P.-CYPRINIF. (Characoid.) Characid.

Copelandellus JORDAN et EVERMANN 1896 : P.-PERCIF. (Percoid.) Etheostomid.

Copelandia JORDAN 1877: P.-PERCIF. (Percoid.) Centrarchid.

Conella MYERS 1956 : P.-CYPRINIF. (Characoid.) Characid.

Cophias FITZINGER 1843: R.SQUAMAT. (Saur.) Tejid.

Cophixalus BOETTEGER 1892: Amph. ANOUR. Microhylid.

Cophophryne BOULENGER 1887: Amph. ANOUR. Bufonid.

Cophoscincus VAILLANT 1884: R.-SQUAMAT. (Saur.) Scincid.

Cophotis PETERS 1861 : R.-SQUAMAT. (Saur.) Agamid.

Cophyla BOETTEGER 1880: Amph. ANOUR. Microhylid.

Cophyxalus : errat. pro Cophixalus BOETTEGER 1892.

Copidoglanis GUNTHER 1864 : P.CYPRINIF. (Siluroid.) Plotosid.

Copiula MEHELY 1901: syn. de Cophixalus BOETTEGER 1892.

Coporhamphus GLOGER 1842 : syn. de Todirhamphus LESSON 1827. 
Copsicus CABANIS 1815 : syn. de Copsychus WAGLER 1827.

Copsychus WAGLER 1827: Av. PASSERIF. (Acromyod.) Turdid. (Phoenicurin.).

Coptodon GERVAIS 1853 : P.-PERCIF. (Percoid.) Cichlid.

Coptostomabarbus DAVID et POLL 1937: P.-CYPRINIF. (Cyprinoid.) Cyprinid.

Copurus STRICKLAND 1841: Av. PASSERIF. (Mesomyod.) Tyrannid. (Fluvicolin.).

Coracia BRISSON 1760: syn. de Pyrrhocorax TUNSTALL 1771.

Coracias LINNE 1758: Av. CORACIADIF. Coraciadid.

Coracina VIEILLOT 1816: Av. PASSERIF. (Mesomyod.) Campephagid.

Coracinus GRONOV 1763 : syn de Dichistius GILL 1888.

Coracodychus LAURENT 1940 : Amph. ANOUR. Ranid.

Coracopitta SCLATER 1888: Av. PASSERIF. (Mesomyod.) Pittid.

Coracopsis WAGLER 1832: Av. PSITTACIF. Psittacid.

Coradion KAUP 1860 : P.-PERCIF. (Percoid.) Chaetodontid.

Coraglanis HORA 1952 : P.-CYPRINIF. (Characoid.) Characid.

Coragyps LE MAOUT 1853: Av. FALCONIF. Vulturid.

Corallicola JORDAN et EVERMANN 1898: syn. de Paraclinus MOCQUARD 1889.

Corallus DAUDIN 1803 : R.-SQUAMAT. (Serpent.) Boid.

Corapipo BONAPARTE 1854: Av. PASSERIF. (Mesomyod.) Piprid.

Corapitta BONAPARTE 1854: Av. PASSERIF. (Mesomyod.) Pittid.

Corcorax LESSON 1830 : Av. PASSERIF. (Acromyod.) Corvid.

Cordorinus RAFINESQUE 1815 : syn. de Corydoras de LACEPEDE 1803.

Cordyloides FRITSCH 1904: R.SQUAMAT. (Saur.) Zonurid.

Cordylosaurus GRAY 1866: R.SQUAMAT. (Saur.) Gerrhosaurid.
Cordylus GRONOV 1763 : R.-SQUAMAT. (Saur.) Zonurid. (serait syn. de Zonurus MERREM $1820)$.

Coreoonus (LINNE 1758) de LACEPEDE 1803 : P.-CLUPEIF. (Salmonoid.) Salmonid.

Coreius JORDAN et STARKS 1905 : P.-CYPRINIF. (Cyprinoid.) Cyprinid.

Corematodus BOULENGER 1897 : P.-PERCIF. (Percoid.) Cichlid.

Coreobagrus MORI 1936: P.-CYPRINIF. (Siluroid.) Bagrid.

Coreoleuciscus MORI 1935 : P.-CYPRINIF. (Cyprinoid.) Cyprinid.

Coreoperca HERZENSTEIN 1896 : P.PERCIF. (Percoid.) Serranid.

Coreosiniperca FANG et CHANG 1932: P.-PERCIF. (Percoid.) Serranid.

Corethruropsis SALVADORI 1875 : Av. RALLIF. Rallid.

Corethura REICHENBACH 1849 : Av. RALLIF. Rallid.

Corica GRAY 1832: P.-CLUPEIF. (Clupeoid.) Clupeid.

Coricus CUVIER 1817 : P.-PERCIF. (Labroid.) Labrid.

Coridodax GUNTHER 1862: P.PERCIF. (Labroid.) Odacid.

Corieus : errat. pro Coreius JORDAN et STARKS 1905.

Coripareius GARMAN 1912 : P.-CYPRINIF. (Cyprinoid.) Cyprinid.

Coriphilus WAGLER 1832: Av. PSITTACIF. Psittacid.

Coris de LACEPEDE 1802 : P.PERCIF. (Labroid.) Labrid.

Corizichthys OGILBY 1908 : P.PERCIF. (Batrachoid.) Batrachoidid.

Corniger AGASSIZ 1831 : P.-BERYCIF. Holocentrid.

Cornix MOEHRING 1758: syn. de Corvus LINNE 1758.

Cornix FORSTER 1817: syn. de Corvus LINNE 1758.

Cornufer TSCHUDI 1838: Amph. ANOUR. Ranid.

Corone KAUP 1829 : syn. de Corvus LINNE 1758.

Coronella LAURENTI 1768: R.SQUAMAT. (Serpent.) Colubrid. 
Coronichthys HERRE 1942: P.PERCIF. (Gobiesoxoid.) Gobiesocid.

Coronogobius HERRE 1945 : P.PERCIF. (Gobioid.) Gobiid.

Corrira BRISSON 1760 : nom. n'Id. pro Aves Incert. sed.

Corsira GRAY 1828 : syn. de Sorex LINNE 1758.

Corthylio CABANIS 1853 : Av. PASSERIF. (Acromyod.) Regulid.

Corucia GRAY 1856 : R.-SQUAMAT. (Saur.) Scincid.

Corusculus JORDAN et SNYDER 1901: P.-PERCIF. (Percoid.) Serranid.

Corvina CUVIER 1829: syn. de Johnius BLOCH 1793.

Corvinella LESSON 1831 : Av. PASSERIF. (Acromyod.) Laniid.

Corvula JORDAN et EIGENMANN 1889: P.-PERCIF. (Percoid.) Sciaenid.

Corvultur LESSON 1831 : Av. PASSERIF. (Acromyod.) Corvid.

Corvus LINNE 1758: Av. PASSERIF. (Acromyod.) Corvid.

Corychthoichthys : errat. pro Corythoichthys KAUP 1856.

Corydalla VIGORS 1825: s./g. d'Anthus BECHSTEIN 1805.

Corydon LESSON nec GLOGER 1842 : Av. PASSERIF. (Desmodactyl.) Eurylaimid.

Corydoras de LACEPEDE 1803 : P.-CYPRINIF. (Siluroid.) Callichthyid.

Corydospiza SUNDEVALL 1872 : Av. PASSERIF. (Acromyod.) Fringillid.

Coryllis FINSCH 1868 : syn. de Loriculus BLYTH 1849.

Corymbonhanes EI GEN M A N 1909 : P.-CYPRINIF. (Siluroid.) Loricariid.

Corynolophus GILL 1879 : P.-LOPHIIF. (Ceratoid.) Ceratiid.

Corynophorus OSORIO 1912 : P.LOPHIIF. (Ceratoid.) Himantolophid.

Corynopoma GILL 1858: P.-CYPRINIF. (Characoid.) Charactd.

Corynorhinus ALLEN 1865 : M.CHIROPT. (Microchiropt.) Vespertilionid. (Vespertilionin.).
Coryornis RIDGWAY 1925 : syn. de Rupornis KAUP 1845.

Corypha GRAY 1840 : Av. PASSERIF. (Acromyod.) Alaudid.

Coryphaena LINNE 1758: P.-CORYPHAENIF. Coryphaenid.

Coryphaenoides GUNNER 1765: P.-GADIF. Macrurid.

Coryphaesopia CHABANAUD 1930: P.-PLEURONECTIF. (Soleioid.) Soleid.

Coryphillus CHABANAUD 1931: P.-PLEURONECTIF. (Soleioid.) Soleid.

Coryphistera BURMEISTER 1860 : Av. PASSERIF. (Mesomyod.) Furnariid.

Coryphoblennius NORMAN 1943 : P.-PERCIF. (Blennioid.) Blenniid.

Coryphoenas WARDLAW-RAISSAY 1890 : syn. de Reinwardtoenas BONAPARTE 1854.

Coryphopterus GILL 1863: P.-PERCIF. (Gobioid.) Gobiid.

Coryphospingus CABANIS 1851: Av. PASSERIF. (Acromyod.) Fringillid.

Coryphotriccus RIDGWAY 1906: Av. PASSERIF. (Mesomyod.) Tyrannid.

Corypithecus TROUESSART 1879 : syn. de Presbytis ESCHSCHOLTZ 1821.

Corythaeola HEINE 1860: Av. CUCULLIF. Musophagid.

Corythaix ILLIGER 1811: syn. de Turacus CUVIER 1800.

Corythaixoides SMITH 1833: Av. CUCULLIF. Musophagid.

Corythobatus CANTOR 1850 : P.PERCIF. (Scorpaenoid.) Scorpaenid.

Corythocichla SHARPE 1833: Av. PASSERIF. (Acromyod.) Timaliid.

Corythoela : errat. pro. Corythaela HEINE 1860.

Corythoichthys KAUP 1856: P.SYNGNATHIF. Synthatid.

Corythomantis BOULENGER 1896: Amph. ANOUR. Hylid.

Corythophanes WAGLER 1830 : R.SQUAMAT. (Saur.) Iguanid. 
Corythopsis (SUNDEVALL 1836) GRAY 1846: Av, PASSERIF. (Mesomyod.) Conopophagid.

Corythornis KAUP 1848 : syn. d'Alcedo LINNE 1758.

Coryzichthys : errat. pro Corizichthys OGILBY 1908.

Cosanoxyron FOWLER 1907 : vide Coscinoxyron FOWLER 1907.

Coscinoxyron FOWLER 1907 : P.CYPRINIF. (Characoid.) Characid.

Coscoroba REICHENBACH 1852 : Av. ANATIF. Anatid. (Anserin.).

Cosmetornis GRAY 1840: Av. CAPRIMLLGIF. Caprimulgid. (Caprimulgin.).

Cosmochilus SAUVAGE 1878: P.CYPRINIF. (Cyprinoid.) Cyprinid.

Cosmonetta (KAUP 1829) GRAY 1855: Av. ANATIF. Anatid. (Anatin.).

Cosmopsarus REICHENOW 1879 : Av. PASSERIF. (Acromyod.) Sturnid.

Cossypha HARTLAUB : Av. PASSERIF. (Acromyod.) Timaliid.

Cossypha VIGORS 1825 : Av. PASSERIF. (Acromyod.) Turdid. (Phoenicurin.).

Cossyphicula GROTE 1934: Av. PASSERIF. (Acromyod.) Turdid. (Phoenicurin.).

Cossyphodes BLEEKER 1860: P.PERCIF. (Labroid.) Labrid.

Cossyphopsis STEJNEGER 1883 : Av. PASSERIF. (Acromyod.) Timaliid.

Cossyphus CUVIER et VALENCIENNES 1839: P.-PERCIF. (Labroid.) Labrid.

Cosymbotus FITZINGER 1843 : R.SQUAMAT. (Saur.) Geckonid.

Cotinga BRISSON 1760: Av, PASSERIF. (Mesomyod.) Cotingid.

Cottapistus BLEEKER 1876 : P.PERCIF. (Scorpaenoid.) Scorpaenid.

Cottinella BERG 1907 : P.-PERCIF. (Cottoid.) Cottid.

Cottiusculus JORDAN et STARKS
1904: P.-PERCIF. (Cottoid.) Cottid.

Cottocomephorus P E L L E G R IN 1901: P.-PERCIF. (Cottoid.) Cottid.

Cottogaster PUTNAM 1863 : P.PERCIF. (Percoid.) Etheostomid.

Cottogobius KOUMANS 1941: P.PERCIF. (Gobioid.) Gobiid.

Cottoperca STEINDACHNER 1876: P.-PERCIF. (Trachinoid.) Nototheniid.

Cottunculoides BARNARD 1927 : P.-PERCIF. (Cottoid.) Cottid.

Cottunculus COLLETT 1875:P.PERCIF. (Cottoid.) Cottid.

Cottus IINNE 1758: P.-PERCIF. (Cottoid.) Cottid.

Coturnicops BONAPARTE 1854 : Av. RALLIF. Rallid.

Coturnix BONNATERRE 1790 : Av. PHASIANIF. Phasianid. (Phasianin.).

Cotyle BOIE 1826 : Av. PASSERIF. (Acromyod.) Hirundinid.

Cotylis MULLER et TROSCHEL 1843 : P. - PER C I F. (Gobiesoxoid.) Gobiesocid.

Cotylopus GUICHENOT 1862: P.PERCIF. (Gobioid.) Gobiid.

Coua OKEN 1817: Av. CUCULLIF. Cucullid.

Couchia THOMPSON 1839 : P.-GADIF. Gadid.

Couesius JORDAN 1878 : P.-CYPRINIF. (Cyprinoid.) Cyprinid.

Cractes BILLBERG 1828 : Av. PASSERIF. (Acromyod.) Corvid.

Cracticus VIEILLOT 1816: Av. PASSERIF. (Acromyod.) Cracticid.

Cranioleuca REICHENBACH 1853 : Av. PASSERIF. (Mesomyod.) Furnariid.

Cranobagrus : P.-CYPRINIF. (Siluroid.) Bagrid.

Cranoglanis PETERS 1880 : P.CY. PRINIF. (Siluroid.) Bagrid.

Cranorhinus CABANIS et HEINE 1860 : Av. CORACIADIF. Bucerotid.

Craspedoglossus MULLER 1922: syn. de Eupsophus FITZINGER 1843. 
Craspedophora GRAY 1840: Av. PASSERIF. (Acromyod.) Paradiseid. (Paradisein.).

Craspedoprion BERLEPSCH et HARTERT 1902: syn. de Rhynchocyclus CABANIS et HEINE 1860 .

Crasseomys MILLER 1900 : syn. de Clethrionomys TILESIUS 1850.

Crassinarke TA GAKI 1951 : P.TORPEDINIF. Torpedinid.

Crassinopsis : Amph. ANOUR. Rhacophorid.

Crater: P.-MUGILIF. Atherinid

Craterocephalus $M c$ CULLOCH 1912 : P.-MUGILIF. Atherinid.

Crateromys THOMAS 1895 : M.-RODENT. (Myomorph.) Murid. (Murin.).

Crateropus SWAINSON 1830:Av. PASSERIF. (Acromyod.) Timaliid. (Turdoidin.).

Crateroscelis SHARPE 1883: Av. PASSERIF. (Acromyod.) Timaliid. (Turdoidin.).

Cratinus STEINDACHNER 1879: P.-PERCIF. (Percoid.) Serranid.

Cratogeomys MERRIAM 1895 : M.RODENT. (Myomorph.) Geomyid.

Cratophopeltis : errat. pro Crotaphopeltis JAN 1863.

Craurothrix THOMAS 1896: syn. d'Echiothrix GRAY 1867.

Cravus RAFINESQUE 1815: syn. de Pyrrhocorax TUNSTALL 1771.

Crax LINNE 1758: Av. PHASIANIF. Cracid.

Crayracion SCHAEFFER 1760 : s./g. de Tetraodon LINNE 1758 .

Creadio CABANIS 1851 : errat. pro Creadion VIEILLOT 1816.

Creadion VIEILLOT 1816 : Av. PASSERIF. (Acromyod.) Corvid.

Creagus BONAPARTE 1854: Av. LARIF. Larid.

Creagrudite MYERS 1927: P.-CYPRINIF. (Characoid.) Characid.

Creagrutops SCHULTZ 1944 : P.CYPRINIF. (Characoid.) Characid.
Creagrutus GUNTHER 1864 : P.CYPRINIF. (Characoid.) Characid.

Creatochanes GUNTHER 1864 : P.CYPRINIF. (Characoid.) Characid.

Creatophora LESSON 1847: syn. de Dilophus VIEILLOT 1816.

Creciscus CABANIS 1856:Av. RALLIF. Rallid.

Crecopsis SHARPE 1893 : Av. RALLIF. Rallid.

Creedia OGILBY 1898 : P.-PERCIF. (Trachinoid.) Creediid.

Creisson JORDAN et SEALE 1907 : P.-PERCIF. (Gobioid.) Gobiid.

Cremnobates GUNTHER 1862 : syn. de Paraclinus MOCQUARD 1889.

Cremnomys WROUGHTON 1912 : syn. de Rattus FISCHER 1803.

Cremnoteleka WHITLEY 1940 : P.-PERCIF. (Blennioid.) Blenniid.

Crenalticus WHITLEY 1930 : P.PERCIF. (Blennioid.) Blenniid.

Crenicichla HECKEL 1840 : $P$.PERCIF. (Percoid.) Cichlid.

Crenichthys HUBBS 1932 : P.-CYPRINODONTIF. Cyprinodontid.

Crenidens CUVIER et VALENCIENNES 1830: P.-PERCIF. (Percoid.) Kyphosid.

Crenilabrus OKEN 1817 : P.-PERCIF. (Labroid.) Labrid.

Crenimugil SCHULTZ 1946 : P.MUGILIF. Mugilid.

Crenophthalmus : $P .-P E R C I F$. (Blennioid.) Blennitd.

Crenuchus GUNTHER 1863: P.CYPRINIF. (Characoid.) Characid.

Creocele BRIGGS 1955: P.-PERCIF. (Gobiesoxoid.) Gobiesocid.

Creolus JORDAN et GILBERT 1882: P.-PERCIF. (Percoid.) Serranid.

Creophaps GOULD: Av. COLUMBIF. Columbid. (Columbin.).

Creotroctes GISTEL 1848 : P.-PERCIF. (Scombroid.) Thunnid.

Crepidogaster GUNTHER 1861 : P.- 
PERCIF. (Gobiesoxoid.) Gobiesocid.

Creurgops SCLATER 1858: Av. PASSERIF. (Acromyod.) Tanagrid.

Crex BECHSTEIN 1803: Av. RALLIF. Rallid.

Cricetiscus THOMAS 1917 : syn. de Phodopus MULLER 1910.

Cricetodivus PEALE 1848 : syn. de Perognathus WIED 1839.

Cricetomys WATERHOUSE 1840 : M.-RODENT. (Myomorph.) Cricetid. (Cricetin.).

Cricetulus MILNE-EDWARDS 1867: M.-RODENT. (Myomorph.) Cricetid. (Cricetin.).

Cricetus LESKE 1779: M.-RODENT. (Myomorph.) Cricetid. (Cricetin.).

Cricosaura PETERS et GUNDLACH $1864: R$. -SQUAMAT. (Saur.) Xanthusiid.

Cridorsa WHITLEY 1938 : P.-PERCIF. (Percoid.) Kyphosid.

Crinia TSCHUDI 1838: Amph. ANOUR. Leptodactylid.

Crinifer JAROCKI 1821: Av. CUCULLIF. Musophagid.

Criniferoides ROBERTS 1926: 4v. CUCULLIF. Musophagid.

Criniger TEMMINCK 1820: Av. PASSERIF. (Acromyod.) Pycnonotid.

Crinodus GILL 1862: P.-PERCIF. (Percoid.) Aplodactylid.

Cristatogobius HERRE 1927: P.PERCIF. (Gobioid.) Gobiid.

Cristemberiza MOMIYAMA 1928: Av. PASSERIF. (Acromyod.) Fringillid. (Emberizin.).

Cristiceps CUVIER et VALENCIENNES 1836 : P.-PERCIF. (Blennioid.) Clinid.

Cristivomer GILL et JORDAN 1878 : P.-CLUPEIF. (Salmonoid.) Salmonid.

Crithagra SWAINSON 1827: Av. PASSERIF. (Acromyod.) Fringillid. (Fringillin.).

Crithagroides : Av. PAS SER IF. (Acromyod.) Fringillid. (Fringillin.).

Crius VALENCIENNES 1843 : P.-
PERCIF. (Stromateoid.) Stromateid.

Crocethia BILLBERG 1828: P.CHARADRIIF. Charadriid. (Scolopacin.).

Crocias TEMMINCK 1836: Av. PASSERIF. (Acromyod.) Timaliid.

Crocidura WAGLER 1832: M.-INSECTIV. Soricid. (Crocidurin.).

Crockeridius CLARK 1936 : P.PERCIF. (Blennioid.) Clinid.

Crocodilurus von SPIX 1825: R.SQLAMAT. (Saur.) Tejid.

Crocodilus (GRONOV 1763) GMELIN 1789 : R.-LORICAT. Crocodilid.

Crocodylus LAURENTI 1768 : syn. de Crocodilus (GRONOV 1763) GMELIN 1789.

Crocomorphus HARGITT 1890 : Av. PICIF. Picid. (Picin.).

Crocopus BONAPARTE 1854: Av. COLUMBIF. Columbid. (Treronin.).

Crocuta KAUP 1828: M.-FISSIP. Hyaennid. (Hyaennin.).

Croilia SMITH 1955: P.-PERCIF. (Gobioid.) Gobiid.

Cromeria BOULENGER 1901 : P.CLUPEIF. (Cromeroid.) Cromeriid.

Cromileptes SWAINSON 1839 : P.PERCIF. (Percoid.) Serranid.

Crossarchus CUVIER 1825 : M.-FISSIP. Viverrid. (Herpestin.).

Crossias JORDAN et STARKS 1904: P.-PERCIF. (Cottoid.) Cottid.

Crossleyia HARTLAUB 1877 : syn. d'Oxylabes SHARPE 1870.

Crossobamon BOETTEGER 1888 : R.-SQUAMAT. (Saur.) Iguanid.

Crossobothus FOWLER $1934: s . / g$. de Bothus RAFINESQUE 1810.

Crossocheilichthys BLEEKER 1860: P.-CYPRINIF. (Cyprinoid.) Cyprinid.

Crossocheilus van HASSELT 1823 : P.-CYPRINIF. (Cyprinoid.) Cyprinid.

Crossochir HUBBS 1933 : P.-PERCIF. (Labroid.) Embiotocid.

Crossodactylodes COCHRAN 1938 : Amph. ANOUR. Bufonid. 
Crossodactylus DUMERIL et BIBRON 1841 : Amp. ANOUR. Bufonid.

Crossoderma GUICHENOT 1869 : P. - PERCIF. (Scorpaenoid.) Scorpaenid.

Crossogale THOMAS 1921 : syn. de Chimmarrogale ANDERSON 1877.

Crossogobius : P. - PERCIF. (Gobioid.) Gobiid.

Crossolepis NORMAN 1927 : syn. de Psettina HUBBS.

Crossoleya : errat. pro Crossleya HARTLAUB 1877.

Crossomys THOMAS 1907 : M.-RODENT. (Myomorph.) Murid. (Hydromyin.).

Crossoptilon HODGSON 1838: Av. PHASIANIF. Phasianid. (Phasianin.).

Crossopus KAUP 1829: syn. de Neomys KAUP 1829.

Crossorhinus MULLER et HENLE 1837 : P. - GALEIF. (Isuroid.) Orectolobid.

Crossorhombus REGAN 1920: P.PLEURONECTIF. (Pleuronectoid.) Bothid.

Crossoscorpaena FOWLER 1938 : P. - PERCIF. (Scorpaenoid.) Scorpaenid.

Crossostoma SAUVAGE 1878: P.CYPRINIF. (Cyprinoid.) Homalopterid.

Crossostomus LAHILLE 1908: errat. pro Crossostoma SAUVAGE 1878.

Crotalinus RAFINESQUE 1818 : syn. de Sistrurus GARMAN 1883.

Crotalophorus GRONOV 1763 : syn. de Sistrurus GARMAN 1883.

Crotalopsis KAUP 1860: P.-ANGUILLIF. (Congroid.) Ophichthyid.

Crotalus LINNE 1758: R.-SQUAMAT. (Serpent.) Crotalid.

Crotaphopeltis JAN 1863 : R.SQUAMAT. (Serpent.) Boigid.

Crotaphytus HOLBROOK 1842 R.-SQUAMAT. (Saur.) Iguanid.

Crotema ROBERTS 1922: Av. CAPRIMULGIF. Caprimulgid. (Caprimulgin.).
Crotophaga LINNE 1758:Av. CUCULLIF. Cucullid.

Crucirostra BREHM 1827 : syn. de Loxia LINNE 1758.

Crunomys THOMAS 1897 : M.-RODENT. (Myomorph.) Murid. (Murin.).

Cruriraja BIGELOW et SCHROEDER 1948 : P.-RAJIF. (Rajoid.) Rajid.

Cruxentina : P.-CYPRINIF. (Characoid.) Characid.

Crydorsa : errat. pro Cridorsa WHITLEY 1938.

Crymnophilus VIEILLOT 1816: Av. CHARADRIIF. Charadriid. (Phalaropin.).

Cryodraco DOLLO 1900: P.-PERCIF. (Trachinoid.) Nototheniid.

Cryptacanthodes STORER 1839: P.-PERCIF. (Blennioid.) Blenniid.

Cryptacanthoides LINDBERG 1930: P.-PERCIF. (Blennioid.) Blenniid.

Cryptigata MATHEWS 1925 : syn. de Phylloscopus BOIE 1826.

Cryptobatrachus RUTHVEN 1916 : Amph. ANOUR. Hylid.

Cryptoblepharus WIEGMAN N 1834 : R. - SQUAMAT. (Saur.) Scincid.

Cryptobranchus LEUCKART 1821: Amph. UROD. Cryptobranchid.

Cryptocentroides POPTA 1922 : P.PERCIF. (Gobioid.) Gobiid.

Cryptocentrus EHRENBERG 1837 : P.-PERCIF. (Gobioid.) Gobiid.

Cryptochloris SHORTRIDGE el CARTER: M. -INSECTIV. Chrysochlorid. (Chrysochlorin.).

Cryptodelma FISCHER 1882: R.SQUAMAT. (Saur.) Pygopodid.

Cryptogale GRANDIDIER 1928: syn. de Geogale MILNE-EDWARDS et GRANDIDIER.

Cryptoglaux RICHEMOND 1901: Av. STRIGIF. Strigid. (Strigin.).

Cryptohylax : Amph. ANOUR. Rhacophorid.

Cryptolestes TATE 1933: syn. de Lestoros OEHSER 1934.

Ann. de Parasitologie, T. XXXVI, No 5-6. -1961. 
Cryptolopha SWAINSON 1837 : Av. PASSERIF. (Acromyod.) Muscicapid.

Cryptolynchus REGAN et TREWAVAS 1932 : P.-LOPHIIF. (Ceratoid.) Linophrynid.

Cryptomys GRAY 1864: M.-RODENT. (Bathyergomorph.) Bathyergid.

Cryptophaps SALVADORI 1893 : Av. COLUMBIF. Columbid. (Columbin.).

Cryptophthalmus FRANZ 1910 : P. - ANGUILLIF. (Anguilloid.) Moringuid.

Cryptophthalmus : P.-SYMBRANCHIF. Symbranchid.

Cryptoprocta BENNETT 1833 : M.FISSIP. Viverrid. (Cryptoproctin.).

Cryptopsaras GILL 1883: P.-LOPHIIF. (Ceratoid.) Ceratiid.

Cryptopterenchelys FOWLER 1925: P. - ANGUILLIF. (Congroid.) Ophichthyid.

Cryptopterichthys BLEEKER 1858: P.-CYPRINIF. (Siluroid.) Silurid.

Cryptopterus (BLEEKER 1858) GUNTHER 1864: P. - CYPRINIF. (Siluroid.) Silurid.

Cryptopterus KAUP 1860 : syn. de Cryptopterenchelys FOWLER 1925.

Cryptopterygium GINSBURG 1951: P.-ANGUILLIF. (Congroid.) Ophichthyid.

Cryptopus DUMERIL et BIBRON 1835 : R. - TESTUDIN. (Thecoph.) Trionychid.

Cryptorhina WAGLER 1827: Av. PASSERIF. (Acromyod.) Corvid.

Cryptoscincus MOCQUARD 1906 : R.SQUAMAT. (Saur.) Scincid.

Cryptosmilia COPE 1869 : P.-PERCIF. (Acanthuroid.) Zanclid.

Crvntospiza SALVADORI 1884 : Av. PASSERIF. (Acromyod.) Ploceid.

Cryntotis GUNTHER 1863: Amph. ANOUR. Leptodactylid.

Cryptotis POMEL 1848 : M.-INSECTIV. Soricid. (Soricin.).

Cryptotomus COPE 1870 : P.-PERCIF. (Labroid.) Scarid.
Cryptotrema GILBERT 1890 : P.PERCIF. (Blennioid.) Clinid.

Crypturellus BRAUBOURNE et CHUBB 1914: Av. TINAMIF. Tinamid.

Crypturornis OBERHOLSER 1922: Av. TINAMIF. Tinamid.

Cryoturus ILLIGER 1811 : syn. de Tinamus LATHAM.

Crystallaria JORDAN et GILBERT 1885: P. - PERCIF. (Percoid.) Etheostomid.

Crystallarias JORDAN et SNYDER 1902: P. - PERCIF. (Cottoid.) Cyclopterid.

Crystallichthys JORDAN et EVERMANN 1898 : P.-PERCIF. (Cottoid.) Cyclopterid.

Crystallodytes FOWLER 1923 : P.PERCIF. (Trachinoid.) Pinguipedid.

Crystallogobius GILL 1863 : P.PERCIF. (Gobioid.) Gobiid.

Crystallogobius HERRE : P.-PERCIF. (Gobioid.) Gobiid.

Ctenerpeton COPE 1897: syn. de Chtonerpeton PETERS 1879.

Ctenichthys HOWELL-RIVERO 1936 : P.-PERCIF. (Percoid.) Cichlid.

Ctenoblepharys TSCHUDI 1845 : R.-SQUAMAT. (Saur.) Iguanid.

Ctenobrycon EIGENMANN 1909 : P.-CY P R I N IF. (Characoid.) Characid.

Ctenochaetus GILL 1884 : P.-PERCIF. (Acanthuroid.) Acanthurid.

Ctenocharax REGAN 1907 : P.-CYPRINIF. (Characoid.) Characid.

Ctenochirichthys REGAN et TREWAVAS 1932 : P.-LOPHIIF. (Ceratoid.) Himantolophid.

Ctenocorissa WHITLEY 1931 : P.PERCIF. (Labroid.) Labrid.

Ctenodactylus GRAY 1828 : M.-RODENT. (Sciuromorph.) Ctenodactylid.

Ctenodon BONAPARTE 1831 : P.PERCIF. (Acanthuroid.) Acanthurid.

Ctenoglyphidodon FOWLER 1919 : P.-PERCIF. (Labroid.) Pomacentrid. 
Ctenogobius GILL 1858 : P.-PERCIF. (Gobioid.) Gobiid.

Ctenolabrus CUVIER et VALENCIENNES 1839: P.-PERCIF. (Labroid.) Labrid.

Ctenolates GUNTHER 1871 : P.PERCIF. (Percoid.) Serranid.

Ctenolucius GILL 1861 : P.-CYPRINIF. (Characoid.) Characid.

Ctenomys de BLAINVILLE 1826 : M. -RODENT. (Caviomorph.) Ctenomyid.

Ctenophallus HERRE 1939 : P.PHALLOSTETHIF. Phallostethid.

Ctenopharyngodon STEINDACHNER 1866: P.-CYPRINIF. (Cyprinoid.) Cyprinid.

Ctenophryne MOCQUARD 1904 : Amph. ANOUR. Microhylid.

Ctenopoma PETERS 1844 : P.-PERCIF. (Anabantoid.) Anabantid.

Ctenopristis ARAMBOURG 1941 : P.-RAJIF. (Rhinobatoid.) Pristid.

Ctenops Mc CLELLAND 1845 : P.PERCIF. (Anabantoid.) Anabantid.

Ctenosaura : cf. Ctenosaurus FITZINGER 1843.

Ctenosaurus FITZINGER 1843 : R.-SQUAMAT. (Saur.) Iguanid.

Ctenoscolopsis FOWLER 1931 : s./g. de Scolopsis CUVIER 1814.

Ctenoscopelus : P.-CLUPEIF. (Myctophoid.) Myctophiid.

Ctenotrypauchen STEINDACHNER 1867: P.-PERCIF. (Gobioid.) Gobiid.

Cualac MILLER 1956: P.-CYPRINODONTIF. Cyprinodontid.

Cubanichthys HUBBS 1926 : P.-CYPRINODONTIF. Cyprinodontid.

Cubiceps LOWE 1843 : P.-PERCIF. (Stromateoid.) Stromateid.

Cucullus LINNE 1758 : Av. CUCULLIF. Cucullid.

Culicicapa SWINHOE 1871: Av. PASSERIF. (Acromyod.) Muscicapid.

Culicivora SWAINSON 1827:Av. PASSERIF. (Mesomyod.) Tyrannid. (Platyrhynchin.).
Culius BLEEKER 1856: P.-PERCIF. (Gobioid.) Eleotrid.

Culter BASILEWSKY 1855 : P.-CYPRINIF. (Cyprinoid.) Cyprinid.

Culticula ABBOTT 1901: P.-CYPRINIF. (Cyprinoid.) Cyprinid.

Cultrichthys SMITH 1938: P.-CYPRINIF. (Cyprinoid.) Cyprinid.

Cultriculus OSHIMA 1919 : P.-CYPRINIF. (Cyprinoid.) Cyprinid.

Cultrops SMITH 1938 : P.-CYPRINIF. (Cyprinoid.) Cyprinid.

Cumbel WHITLEY 1952 : P.-PERCIF. (Scorpaenoid.) Platycephalid.

Cuncuma HODGSON 1837: Av. FALCONIF. Falconid. (Aquilin.).

Cuniculus BRISSON 1762 : M.-RODENT. (Caviomorph.) Cuniculid.

Cuniculus MEYER 1790: syn. d'Oryctolagus LILLJEBORG 1874 .

Cunningtonia BOULENGER 1906 : P.-PERCIF. (Percoid.) Cichlid.

Cuon HODGSON 1838 : M.-FISSIP. Canid. (Simocyonin.).

Cuora GRAY 1855 : R.-TESTUDIN. (Thecoph.) Emydid.

Cuphopterus HARTLAUB 1866 : Av. PASSERIF. (Acromyod.) Prionopid.

Cupidonia REICHENBACH 1852 : Av. APODIF. Trochilid.

Curimata WALBAUM 1792: vide Curimatus OKEN 1817.

Curimatella EIGENMANN et EIGENMANN 1889: P. - CYPRINIF. (Characoid.) Characid.

Curimatichthys : P.-CYPRINIF. (Characoid.) Characid.

Curimatoides FOWLER 1940 : P.CYPRINIF. (Characoid.) Characid.

Curimatonsis STEINDACHNER 1876: P. - CYPRINIF. (Characoid.) L'haracid.

Curimatorbis : P.-CYPRINIF. (Characoid.) Characid.

Curimatus OKEN 1817 : P.-CYPRINIF. (Characoid.) Characid. 
Curioptera WHITLEY 1951: P.PLEURONECTIF. (Pleuronectoid.) Pleuronectid.

Curruca MOEHRING 1758 : syn. de Sylvia SCOPOLI 1769.

Currupiscis WHITLEY 1931 : P.PERCIF. (Scorpaenoid.) Triglid.

Cursonia : Av. PASSERIF. (Acromyod.) Timalizd.

Cursorius LATHAM 1790: Av. CHARADRIIF. Clareolid.

Cursus SCLATER: Av. PASSERIF. (Acromyod.) Icterid. (Agelaein.).

Curtipenis RIVAS et MYERS 1950 : P.-CYPRINIF. Poeciliid.

Curucujus BONAPARTE 1854 : Av. TROGONIF. Trogonid.

Curupiscis : cf. Curupiscis WHITLEY 1931.

Cutia HODGSON 1837 : Av. PASSERIF. (Acromyod.) Timaliid.

Cuvierius GRAY 1866 : syn. de Balaenoptera de $L A C E P E D E$ 1804.

Cyanalcyon BONAPARTE 1854: Av. CORACIADIF. Alcedinid.

Cyananthus REICHENBACH 1854 : Av. APODIF. Trochilid.

Cyanecula BREHM 1828: syn. de Cyanosylvia.

Cyanerpes OBERHOLSER 1899 Av. PASSERIF. (Acromyod.) Coerebid.

Cvanicterus BONAPARTE 1850: Av. PASSERIF. (Acromyod.) Icterid.

Cyanocephalus BONAPARTE 1840 : Av. PASSERIF. (Acromyod.) Corvid.

Cyanochen BONAPARTE 1856:Av. ANATIF. Anatid. (Anserin.).

Cyanocincla HUME $1873: s . / g$. de Monticola BOIE 1822.

Cyanocitta STRICKLAND 1845 : Av. PASSERIF. (Acromyod.) Corvid.

Cvanocompsa CABANIS 1861: Av. PASSERIF. (Acromyod.) Fringillid.

Cyanocorax BOIE 1826 : Av. PASSERIF. (Acromyod.) Corvid.

Cyanodacnis CASSIN 1864: Av. PASSERIF. (Acromyod.) Coerebid.
Cyanoderma SALVADORI 1874: syn. de Stachyris HODGSON 1844.

Cyanodialossa CASSIN 1864: Av. PASSERIF. (Acromyod.) Coerebid.

Cyanogarrulus BONAPARTE 1850: Av. PASSERIF. (Acromyod.) Corvid.

Cyanograucalus HARTLAUB 1861: Av. PASSERIF. (Acromyod.) Campepnagid.

Cyanolaemus STONE 1907: Av. APODIF. Trochilid.

Cyanolanius BONAPARTE 1854: Av. PASSERIF. (Acromyod.) Laniid.

Cyanolesbia STEJNEGER 1885 : Av. APODIF. Trochilid.

Cyanolimnas BARBOUR et PETERS 1927: Av. RALLIF. Rallid.

Cyanoloxia BONAPARTE 1850 : Av. PASSERIF. (Acromyod.) Fringillid. (Fringillin.).

Cyanolyca CABANIS 1851: Av. PASSERIF. (Acromyod.) Corvid.

Cyunolyseus BONAPARTE 1854: Av. PSITTACIF. Psittacid.

Cyanomitra REICHENBACH 1853 : Av. PASSERIF. (Acromyod.) Nectariniid.

Cyanomyia BONAPARTE 1854:Av. APODIF. Trochilid.

Cyanomyias SHARPE 1879: Av. PASSERIF. (Acromyod.) Muscicapid.

Cyanophaia REICHENBACH 1854 : Av. APODIF. Trochilid.

Cyanophasis BUTURLIN 1908 : Av. PHASIANIF. Phasianid. (Phasianin.).

Cyanopica BONAPARTE 1850: Av. PASSERIF. (Acromyod.) Corvid.

Cyanopitta GOULD 1880 : Av. PASSERIF. (Mesomyod.) Pittid.

Cyanopolius BONAPARTE 1850 : Av. PASSERIF. (Acromyod.) Corvid.

Cyanops BONAPARTE 1854:Av. PASSERIF. (Acromyod.) Corvid. 
Cyanonsitta BONAPARTE 1854: Av. PSITTACIF. Psittacid.

Cyanonsittacus SALVADORI 1891 : emend. pro Cyanopsitta B. 1854.

Cyanontila BLYTH 1847 : Av. PASSERIF. (Acromyod.) Muscicapid.

Cyanorhamphus BONAPARTE 1854: Av. PSITTACIF. Psittacid.

Cyanospiza BAIRD 1858 : Av. PASSERIF. (Acromyod.) Fringillid. (Fringillin.).

Cyanosylvia BREHM 1828: Av. PASSERIF. (Acromyod.) Turdid. (Phoenicurin.).

Cyanotis SWAINSON 1837: Av. PASSERIF. (Mesomyod.) Tyrannid. (Spiziornithin.).

Cvanotreron BONAPARTE 1854 : Av. COL UMBIF. Columbid. (Treronin.).

Cyathochromis TREWAVAS 1935 : P.-PERCIF. (Percoid.) Cichlid.

Cyathonharynx REGAN 1920 : P.PERCIF. (Percoid.) Cichlid.

Cybiosarda WHITLEY $1935:$ s./g. de Scombreromorus.

Cybirhynchus : Av. PASSERIF. (Desmodactyl.) Eurylaimid.

Cybium CUVIER 1829: syn. de Scombreromorus.

Cyclagras COPE 1885 : R.-SQUAMAT. (Serpent.) Colubrid.

Cyclanorbis GRAY 1852: R.-TESTUDIN. (Thecoph.) Trionychid.

Cyclarhis SWAINSON 1824: Av. PASSERIF. (Acromyod.) Vireonid.

Cyclemys BELL 1834: R.-TESTUDIN. (Thecoph.) Emydid.

Cycleptus RAFINESQUE 1819 : P.CYPRINIF. (Cyprinoid.) Catostomid.

Cyclichthys KAUP 1855: P.-TETRAODONTIF. (Tetraodontoid.) viodontid.

Cyclocheilichthys BLEEKER 1859 : P.-CYPRINIF. (Cyprinoid.) Cyprinid.

Cyclocorus DUMERIL 1853: R.SQUAMAT. (Serpent.) Colubrid.
Cvcloderma PETERS 1854 : R.TESTUDIN. (Thecoph.) Trionychid.

Cyclodus WAGLER 1828: R.-SQUAMAT. (Saur.) Scincid.

Cvelogaster GRONOV 1760 : P.PERCIF. (Scorpaenoid.) Cyclogasterid.

Cyclolumpus TANAKA 1912 : P.PERCIF. (Cottoid.) Cyclopterid.

Cyclonarce GILL 1861 : P.-TORPEDINIF. Torpedinid.

Cyclopes GRAY 1821: M.-EDENT. (Xenerthr.) Myrmecophagid.

Cyclopharynx : P.-PERCIF. (Percoid.) Cichlid.

Cyclophiops BOULENGER 1888: R.-SQUAMAT. (Saur.) Lacertid.

Cyclonhis G UN THE R 1858 : R.SQUAMAT. (Serpent.) Colubrid.

Cyclopium SWAINSON 1838: P.CYPRINIF. (Siluroid.) Loricariid.

Cyclops : errat. pro Cyclopes GRAY 1821.

Cyclopsetta GILL 1889 : P.-PLEURONECTIF. (Pleuronectoid.) Bothid.

Cyclopsis POPOV 1931: P.-PERCIF. (Cottoid.) Cyclopterid.

Cyclopsitta REICHENBACH 1850 : Av. PSITTACIF. Psittacid.

Cyclospittacus SUNDEVALL 1872 : emend. pro Cyclopsitta R. 1850.

Cyclopterichthys STEINDACHNER 1881 : P.-PERCIF. (Cottoid.) Cyclopterid.

Cyclopterocottus POPOV 1930 : P.-PERCIF. (Cottoid.) Cyclopterid.

Cyclopteroides GARMA N 1892 : P.-PERCIF. (Cottoid.) Cyclopterid.

Cyclopteropsis SOLDATOV et POPOV 1929 : P.-PERCIF. (Cottoid.) Cyclopterid.

Cyclopterus LINNE 1758 : P.-PERCIF. (Cottoid.) Cyclopterid.

Cyclorana STEINDACHNER 1867 : Amph. ANOUR. Leptodactylid.

Cyclorhamphus TSCHUDI 1838: nom. nud. syn. de Telmatobius WIEGMANN 1835. 
Cyclorhamphus DUMERIL: Amph. ANOUR. Leptodactylid.

Cyclorhamphus BOULENGER : syn. de Grypiscus COPE 1867.

Cyclorhina PETERS 1871: syn. d'Hipposideros GRAY 1831.

Cyclorhis SWAINSON 1827: emend. pro Cuclarhis SWAINSON 1824.

Cyclorhynchus KAUP 1829: Av. ALCIF. Alcid. (Fraterculin.).

Cyclothone GOODE 1882 : P.-CLUPEIF. (Stomiatoid.) Gonostomid.

Cycloturus GRAY 1825: syn. de Cyclopes GRAY 1821.

Cyclura HARLAN 1825: R.-SQUAMAT. (Saur.) Iguanid.

Cyema GUNTHER 1878: P.-ANGUILLIF. (Nemichthyoid.) Cyemid.

Cygnodraco WAITE 1916 : P.-PERCIF. (Trachinoid.) Nototheniid.

Cygnopsis BRANDT 1836 : A. ANATIF. Anatid. (Anserin.).

Cygnus BARTRAM 1791: Av. ANATIF. Anatid. (Anserin.).

Cylindrophis WAGLER 1828: R.SQUAMAT. (Serpent.) Ilysiid.

Cylindrosteus RAFINESQUE 1820 : P.-AMIIF. Lepisosteid.

Cymatogaster GIBBONS 1854: P.-PERCIF. (Labroid.) Embiotocid.

Cymbacephalus FOWLER 1938: P.-PERCIF. (Scorpaenoid.) Platycephaud.

Cymbilaimus GRAY 1840: Av. PASSERIF. (Mesomyod.) Formicariid. (Thamnophilin.).

Cymbylanius SCLATER 1855: errat. pro Cymbilaimus GRAY 1840.

Cymborhynchus AGASSIZ 1846: Av. PASSERIF. (Desmodactyl.) Eurylaimid.

Cymnocorymbus : P.-CYPRINIF. (Characoid.) Characid.

Cymochorea COUES 1864: Av. PROCELLARIIF. Hydrobatid.

Cymodroma RIDGWAY 1884 : syn. de Fregetta BONAPARTE 1856.

Cymolutes GUNTHER 1861: P.PERCIF. (Labroid.) Labrid.
Cynaedus GRONOV 1763 : P.-PERCIF. (Labroid.) Labrid.

Cynaelurus GLOGER 1841: syn. d'Acinonyx BROOKES 1828.

Cynailurus WAGLER 1830: syn. de Acinonyx BROOKES 1828.

Cynalopex SMITH 1839: syn. de Vulpes OKEN 1816.

Cynamolgus REICHENBACH 1862 : syn. de Macaca de LACEPEDE 1799.

Cynanthus : SWAINSON 1827: Av. PASSERIF (Acromyod.) Motacillid.

Cynchramus BOIE 1826: syn. d'Emberiza LINNE 1758.

Cyneichthys $O$ G I L B Y 1910 : P.PERCIF. (Blennioid.) Blenniid.

Cynias GILL 1903 : syn. de Mustelus LINCK.

Cynichthys SWA INSON 1839 : P.-PERCIF. (Percoid.) Serranid.

Cynicoglossus BONAPARTE 1837 : P.-PLEURONECTIF. (Pleuronectoid.) Pleuronectid.

Cynictis OGILBY 1833 : M.-FISSIP. Viverrid. (Herpestin.).

Cynocephalus (KLEIN) SCHAEFFER 1760 : P.-GALEIF. (Carcharhinoid.) Scyliorhinid.

Cynocephalus BODDAERT 1768: M.-DERMOPTER. Cynocephalid.

Cynocephalus CUVIER et GEOFFROY ST-HILAIRE 1795: syn. de Papio MULLER 1773.

Cynocharax FOWLER 1907 : P.-CYPRINIF. (Characoid.) Characid.

Cynodium : s./g. d'Anthus BECHSTEIN 1805.

Cynodon SPIX 1829: nom. nud. pro Rhaphiodon L. AGASSIZ 1829.

Cynodontichthys MEEK 1904: P.CYPRINODONTIF, Poeciliid.

Cynodontophis WERNER 1902: syn. de Miodon DUMERIL 1859.

Cynofelis LESSON 1842: syn. de Acinonyx BROOKES 1828.

Cynogale LUND 1842: syn. de Speothos LUND 1839. 
Cynogale GRAY 1837 : M.-FISSIP. Viverrid. (Hemigalin.).

Cynoglossoides BONDE 1923 : P.PLEURONECTIF. (Soleoid.) Cynoglossid.

Cynoglossus HAMILTON-BUCHANAN 1822: P.-PLEURONECTIF. (Soleoid.) Cynoglossid.

Cynolebias STEINDACHNER 1877 : P.-CYPRINODONTIF. Cyprinodontid.

Cynomacaca KHAJURIA 1953 : M.PRIMAT. (Simioid.) Cercopithecid. (Cercopithecin.).

Cynomacrurus DOLLO 1909: P. GADIF. Macrurid.

Cynomolgus TROUESSART 1904: errat. pro Cynamolgus REICHENBACH 1862.

Cynomops THOMAS $1920: s . / g$. de Molossops PETERS 1865.

Cynomys RAFINESQUE 1817 : M.RODENT. (Sciuromorph.) Sciurid. (Sciurin.).

Cynonycteris PETERS 1852: syn. de Roussettus GRAY 1821.

Cynopanchax AHL 1928: P.-CYPRINODONTIF. Cyprinodontid.

Cynoperca GILL et JORDAN 1877 : P.-PERCIF. (Percoid.) Percid.

Cynophidium REGAN 1914 : P.PERCIF. (Ophidioid.) Brotulid.

Cynophis GRAY 1849: R.-SQUAMAT. (Serpent.) Colubrid.

Cynopithecus GEOFFROY ST-HILAIRE 1835 : M.-PRIMAT. (Simioid.) Cercopithecid. (Cercopithecin.).

Cynopoecilus REGAN 1912: s./g. de Pterolebias GARMAN 1895.

Cynoponticus COSTA 1845 : P.-ANGUILLIF. (Congroid.) Congrid.

Cynopotamus CUVIER et VALENCIENNES 1849 : P.-CYPRINIF. (Characoid.) Characid.

Cynops TSCHUDI 1838: Amph. UROD. Salamandrid.

Cynopsetta JORDAN et STARKS 1906 : P. - PLEURONECTIF. (Pleuronectoid.) Pleuronectid.

Cynopterus CUVIER 1824: M.CHIROPT. (Megachiropt.) Pteropid. (Pteropin.).
Cynorhinus ALLEN : M.-CHIROPT. (Microchiropt.) Vespertilionid. (Vespertilionin.).

Cynoscartes NORMAN $1943: s . / g$. de Scarichthys BLEEKER 1861.

Cynoscion GILL 1861 : P.-PERCIF. (Percoid.) Sciaenid.

Cynothrissa REGAN 1917 : P.-CLUPEIF. (Clupeioid.) Clupeid.

Cynotilapia REGAN 1921 : P.-PERCIF. (Percoid.) Cichlid.

Cyon BLANFORD 1888: emend. pro Cuon HODGSON 1838.

Cyornis BLYTH 1843 : Av. PASSERIF. (Acromyod.) Muscicapid.

Cypho MYERS 1940 : P.-PERCIF. (Percoid.) Serranid.

Cyphocharax FOWLER 1906 : P.CYPRINIF. (Characoid.) Characid.

Cyphomycter FOWLER et BEAN 1929: P. - PERCIF. (Acathuroid.) Acanthurid.

Cyphorina LESSON 1843: Av. PASSERIF. (Acromyod.) Troglodytid.

Cyphorhinus CABANIS 1844: Av. PASSERIF. (Acromyod.) Troglodytid.

Cyphoscopelus F O WLE 1925 : P.-CLUPEIF. (Myctophoid.) Myctophiid.

Cyphotilapia $R E G A N$ 1920: P.PERCIF. (Percoid.) Cichlid.

Cyprichthys WHITLEY 1936 : P.TETRAODONTIF. (Tetraodontoid.) Tetraodontid.

Cyprinella GIRARD $1856: s . / g$. de Notropis RAFINESQUE 1818.

Cyprinion HECKEL 1843 : P.-CYPRINIF. (Cyprinoid.) Cyprinid.

Cyprinocirrhites TANAKA 1918: P.-PERCIF. (Percoid.) Cirrhitid.

Cyprinodon de LACEPEDE 1803 : P.-CYPRINODONTIF. Cyprinodontid.

Cyprinogobius KOUMANS 1937: P.-PERCIF. (Gobioid.) Gobiid.

Cyprinopsis FITZINGER 1832 : P.CYPRINIF. (Cyprinoid.) Cyprinid. 
Cyprinus LINNE 1758: P.-CYPRINIF. (Cyprinoid.) Cyprinid.

Cypselichthys STEINDACHNER 1883 : P. - PERCIF. (Stromateoid.) Stromateid.

Cypseloides STEUBEL 1848: Av. APODIF. Apodid.

Cypselurus SW AINSON 1839 : P.-BELONIF. (Exoxoetoid.) Exocoetid.

Cypselus I L L I GER 1811: syn. d'Apus SCOPOLI 1777.

Cypsirhina VIEILLOT 1816: Av. PASSERIF. (Acromyod.) Corvid.

Cypsiurus LESSON 1843 : Av. APODIF. Apodid.

Cypsnagra LESS ON 1831: Av. PASSERIF. (Acromyod.) Tanagrid.

Cyrene HECKEL 1843 : P.-CYPRINIF. (Cyprinoid.) Cyprinid.

Cyromys THOMAS 1910: syn. d'Uromys PETERS 1867.

Cyrrhitops SMITH 1951: P.-PERCIF. (Percoid.) Cirrhitid.

Cyrtocara BOULENGER 1902 : P.PERCIF. (Percoid.) Cichlid.

Cyrtocharax FOWLER 1906 : P.-CYPRINIF. (Characoid.) Characid.

Cyrtodactylus GRAY 1827: R.SQUAMAT. (Saur.) Geckonid.
Cyrtonyx GOULD 1844 : Av. PHASIANIF. Phasianid. (Phasianin.).

Cyrtophis SMITH 1849 : syn. d'Aspidelaps FITZINGER 1843.

Cyrtorhynchus COSTA 1855 : P.CLUPEIF. (Myctophoid.) Myctophiid.

Cyrtostomus CABANIS 1851: Av. PASSERIF. (Acromyod.) Nectariniid.

Cyrtus AGASSIZ 1846 : vide Kurtus BLOCH 1786.

Cystignathus WAGLER 1830 : Amph. ANOUR. Leptodactylid.

Cystophoca BRASS 1911: syn. de Cystophora NILSSON 1820.

Cystophora NILS S O 1820 : M.PINNIP. Phocid. (Cystophorin.).

Cyttoides WE T T S T E IN 1887 : P.-ZEIF. Zeid.

Cyttomimus GILBERT 1905: P.-ZEIF. Zeid.

Cyttopsis GILL 1862: P.-ZEIF. Zeid.

Cyttosoma GILCHRIST 1904: P.ZEIF. Zeid.

Cyttula WEBER 1913: P.-ZEIF. Zeid.

Cyttus GUNTHER 1860 : P.-ZEIF. Zeid.

\section{ERRATA}

Errata pour le n* 3 (Trentenaire de la Station de Richelieu), ces Annales, tome XXXVI, 1961 :

p. 186, lignes 2 et 7, après F.G.A.M. Smit, ajouter : 1955.

p. 329, ligne $10 \quad$ id. id.

p. 226, ligne 14, et p. 336, dernière ligne, remplacer Colynisus par Leptosphyra.

Par suite d'une omission lors de la mise en pages, les lettres majuscules indicatrices des sections du chapitre II n'ont pas été imprimées. En outre, la section N, Diptères piqueurs de Mammifères, qui devait figurer p. 334 avant «Cératopogonides », et la section $\mathrm{O}$, Diptères parasites de Batraciens, ont été supprimées. Dans cette dernière section, il y a seulement une espèce : Forcipomyia (Lasiohelea) velox Winnertz mentionnée aux pages 235,258 et 312 . 